\title{
Weak Three Dimensionality of a Flow Around a Slender Cylinder: the Ginzburg-Landau Equation
}

\author{
J.A.P.Aranha \\ NDF, Mech. Eng, EPUSP \\ S. P, Brazil \\ japaran@usp.br
}

In this paper a weak three-dimensionality of the flow around a slender cylinder is considered and the related model, the so-called Ginzburg-Landau equation, is here obtained as an asymptotic solution of the $3 D$ (discrete) Navier-Stokes equation. The derivation is in line with existing slender bodies theories, as the Lifting Line Theory, for example, where the basic $2 D$ flow, leading to Landau's equation, is influenced now by a "sidewash" that modifies bi-dimensionally the original flow through mass conservation. The theory is asymptotically consistent and rests on an assumption that holds in the vicinity of the Hopf bifurcation (Recr $\approx 45)$; furthermore, it leads to a well-established way to determine numerically both the Landau's coefficient $\mu$ and Ginzburg's coefficient $\gamma$. Arguments are given suggesting that this assumption should hold far beyond Hopf bifurcation (Re $>>$ Recr) and, with it, to extend the Ginzburg-Landau equation almost to the border of the transition region $R e \approx 105$. In this work only the theoretical development is addressed; numerical results will be presented in a forthcoming paper.

Keywords: Hydrodynamic stability, Ginzburg-Landau equation, slender cylinder

\section{Introduction}

Viscous flow around a 2D circular cylinder is known to produce spontaneous harmonic oscillations of the wake for Reynolds number above a critical value $\mathrm{Re}_{\mathrm{cr}} \approx 45$, the oscillating part of the pressure giving rise to an harmonic transverse force on the cylinder that has importance in several engineering applications. It seems to be now well established that this is essentially a stability problem, see Huerre \& Monkewitz (1990), and that the oscillatory wake can be identified with Hopf birfucation in the language of the dynamic systems theory: this idea was first advanced, in a more operational way, by Bishop \& Hassan (1964) and it has been verified experimentally, among others, by Provansal et. al. (1987) in their study in the vicinity of the critical Reynolds number. ${ }^{1}$

Empirical evidences show that both the shedding frequency (Strouhal number) and the whole phenomenon of the vortex induced vibration, at least in its more macroscopic appearance, are essentially invariant with Reynolds number up to the transition zone $\left(\operatorname{Re} \approx 10^{5}\right)$. This observation has led some authors (Iwan \& Blevins (1975), for example) to propose (heuristic) phenomenological models, based on Van der Pol equation, to predict the hydro-elastic interaction, with results that are impressive given the somewhat loose fluid dynamic background on which they are based; interestingly enough, the predictions from such models are, in some aspects, in much better agreement with experiments than the ones obtained from direct CFD computation. Being heuristic as they are, however, they can be used only as interpolators and hardly to extrapolate results to situations much beyond the empirical data on which they are based; furthermore, some hysteric behavior observed in the experiments are not recovered by these models and, obviously, the direct link with the Navier-Stokes equation is lacking in such approach.

The final purpose of the on-going research is to derive a "fluidelastic oscillator model" directly from Navier-Stokes equation, rendering it not only whole predictive but also making it possible to be used in different situations from the ones observed in the existing experimental facilities; in particular, the case where the incident current changes both in direction and intensity along the cylinder span is particularly relevant for offshore applications. Notice also

Presented at ENCIT2004 - 10th Brazilian Congress of Thermal Sciences and Engineering, Nov. 29 -- Dec. 03, 2004, Rio de Janeiro, RJ, Brazil. Technical Editor: Atila P. Silva Freire. that the link with the more fundamental Navier-Stokes equation has here an even greater motivation, since direct computation with CFD did not produce yet a reliable result.

In the present paper only the first step towards this final goal is addressed, namely, to derive the "fluid oscillator model" by considering the cylinder fixed in the flow. The model is represented by the so-called Ginzburg-Landau Equation, see (3.1a), first proposed by Albarède et al (1990) in the context of VIV, with a basic difference, however, in relation to the usual approach: now this equation is not fitted externally to the problem but it results from a consistent asymptotic approximation of the 3D (discrete) Navier-Stokes Equation (NSE). In particular, the coefficients of this equation - the Landau's coefficient $\mu$ and the Ginzburg's coefficient $\gamma-$ are not inferred from the experiments but they are directly computed by well established numerical procedures based on the Finite Element Method (FEM) applied to the 2D cross-flow problem: as usual in "slender bodies theories", one has thus an essentially $2 \mathrm{D}$ effort to compute a $3 \mathrm{D}$ result.

The discrete FEM model is derived, as always, in a finite fluid region $\mathrm{R}$ and one has certainly a difficulty to define the "discrete fluid flow operator" in $\mathrm{R}$ due to the loosely known form of the proper boundary condition at the outlet of $\mathrm{R}$. By considering the flow equation in the wake it has been possible to express the "resistance" offered by the wake on the flow within R, named here the "wake impedance", by an explicit expression that depends solely on the velocity and acceleration of the flow on the borderline that defines the interface between $\mathrm{R}$ and the wake. This derivation is elaborated elsewhere and it may have an importance that transcends the specific application aimed in this work.

The final discrete NSE emulates the continuum NSE with a local inertia, a convective inertia and a viscous dissipation that incorporate the contributions from both the finite fluid region $\mathrm{R}$, that is actually discretized, and the wake. This discrete set of equations are thus projected into the solenoidal and gradient sub-spaces and standard results in Linear Algebra are used to show the inner consistency of these projections; in particular, the projection on the solenoidal sub-space, that determines the velocity field, leads to a normal quadratic dynamic system to which the usual asymptotic procedure can be applied, to determine first Landau's Equation in the 2D context and after the 3D Ginzburg-Landau Equation.

As it is known, a myriad of interesting small scale features, some of them uncovered by a detailed numerical analysis, appear concomitantly with the gross macroscopic ordered behavior of the wake that really matters in the study of the hydro-elastic phenomenon (VIV): the purpose in this work it is not, thus, to 
present a taxonomy of the chaos but rather to capture the underlying order. To achieve this goal a sort of "blindness" is needed, to avoid a too detailed picture, and the asymptotic theory is just a technical filter that provides it. This theory rests on a well defined assumption, see (2.21), that can be directly verified by the numerical results; furthermore, although strictly justified only in the vicinity of Hopf bifurcation $\left(\operatorname{Re} \approx \mathrm{Re}_{\mathrm{cr}}\right.$ ), it seems to hold in a much broader range of Reynolds numbers, what makes possible to extend Ginzburg-Landau equation to this range. Incidentally, this yet speculative result can furnish a theoretical background for the socalled "phenomenological models" that are in fact applied, with a relative success in the prediction of VIV, in a range of Reynolds numbers far beyond the Hopf bifurcation ( $\left.\operatorname{Re}>>\operatorname{Re}_{\mathrm{cr}}\right)$.

The paper is organized as follows: in section 2 the twodimensional problem is addressed, leading to Landau's equation, and in section 3 a weak three dimensionality of the flow is considered and Ginzburg-Landau equation is obtained. Some more technical results, including the derivation of the "wake impedance", are derived elsewhere and numerical results will be presented in a forthcoming paper.

\section{Two-Dimensional Solution: Landau's Equation}

In this section the two dimensional cross-flow around a cylinder is considered. Points in the cross section plane are designated by the vector $\mathbf{x}=\mathrm{xi}+\mathrm{y} \mathbf{j}$, the fluid velocity by the vector field $\mathbf{u}(\mathbf{x}, \mathbf{t})=$ $\mathrm{u}(\mathbf{x}, \mathrm{t}) \mathbf{i}+\mathrm{v}(\mathbf{x}, \mathrm{t}) \mathbf{j}$, the pressure by the function $\mathrm{p}(\mathbf{x}, \mathrm{t})$ while the differential operator $\nabla$ is defined by the expression $\nabla=\mathbf{i} \partial / \partial \mathbf{x}+$ $\mathbf{j} \partial / \partial \mathrm{y}$; these notations will be kept all through the work, even in the next section where the three dimensional correction will be addressed.

Let $d=1$ be the typical dimension of the cylinder cross section (the cylinder diameter in the case of a circular cylinder) and $U=1$ be the incident velocity along the $\mathrm{x}$-axis at the infinite; obviously, the non-dimensional frequency $\omega d / \mathrm{U}$ coincides numerically with $\omega$ and both forms will be used here, depending on the convenience. The fluid density will be also assumed unitary $(\rho=1)$ and thus $\mu_{v}=$ $\mu_{\mathrm{v}} / \rho \mathrm{U} d=1 / \operatorname{Re}$, where $\mu_{\mathrm{v}}$ is the fluid viscosity and Re the Reynolds number.

It is desirable to work here with a velocity field $\mathbf{u}(\mathbf{x}, \mathrm{t})$ that satisfies homogeneous boundary condition both at the infinite and at the cross section contour line $\partial \mathrm{B}$. With this purpose in mind one introduces here an auxiliary vector field $\mathbf{u}_{\mathrm{p}}(\mathbf{x})$ such that

$$
\begin{aligned}
& \bullet \nabla \cdot \mathbf{u}_{\mathrm{p}}=0 ; \\
& \left.\bullet \mathbf{u}_{\mathrm{p}}(\mathbf{x})\right|_{\mathbf{x} \in \partial \mathrm{B}}=\mathbf{0} ; \\
& -\lim _{|\mathbf{x}| \rightarrow \infty} \mathbf{u}_{\mathrm{p}}(\mathbf{x})=\mathbf{i},
\end{aligned}
$$

with the subsidiary condition that $\mathbf{u}_{\mathrm{p}}(\mathbf{x})$ approaches its limit value $\mathbf{i}$ "fast enough", namely: $\mathbf{u}_{\mathrm{p}}(\mathbf{x}) \cong \mathbf{i}$ for $|\mathbf{x}|>5 d$, for example. In the case of a circular cross section this field can be determined with the help of the stream function

$$
\begin{aligned}
& \psi_{\mathrm{p}}(\mathbf{x})=-\mathrm{r} \sin \theta+a_{1} \cdot \frac{\sin \theta}{\mathrm{r}^{\alpha-1}}+a_{2} \cdot \frac{\sin \theta}{\mathrm{r}^{\alpha}} \\
& a_{1} / \mathrm{r}_{\mathrm{c}}^{\alpha}=\alpha+1 ; a_{2} / \mathrm{r}_{\mathrm{c}}^{\alpha+1}=-\alpha ;\left(\mathrm{r}_{\mathrm{c}}=1 / 2\right),
\end{aligned}
$$

where $\mathbf{u}_{\mathrm{p}}(\mathbf{x}) \cong \mathbf{i}$ for $|\mathbf{x}|>5 d$ with an error smaller than $0.025 \%$ for $\alpha$ $=5$. If the cross section is arbitrary this function $\mathbf{u}_{\mathrm{p}}(\mathbf{x})$ can be determined numerically, for instance, but once this is done the actual velocity field $\mathbf{u}_{\mathrm{T}}(\mathbf{x}, \mathrm{t})$ can be written as

$$
\mathbf{u}_{\mathrm{T}}(\mathbf{x}, \mathrm{t})=\mathbf{u}_{\mathrm{p}}(\mathbf{x})+\mathbf{u}(\mathbf{x}, \mathrm{t}) .
$$

Introducing now the (volume) force vector

$$
\mathbf{f}_{\mathrm{p}}(\mathbf{x})=-\left(\mathbf{u}_{\mathrm{p}} \cdot \nabla\right) \mathbf{u}_{\mathrm{p}}+\frac{1}{\operatorname{Re}} \nabla^{2} \mathbf{u}_{\mathrm{p}},
$$

the flow problem is reduced to determine the fields $\{\mathbf{u}(\mathbf{x}, \mathrm{t}) ; \mathrm{p}(\mathbf{x}, \mathrm{t})\}$ such that

$$
\begin{aligned}
& \bullet \frac{\partial \mathbf{u}}{\partial \mathrm{t}}-\frac{1}{\operatorname{Re}} \nabla^{2} \mathbf{u}+\left[\left(\mathbf{u}_{\mathrm{p}} \cdot \nabla\right) \mathbf{u}+(\mathbf{u} \cdot \nabla) \mathbf{u}_{\mathrm{p}}\right]+(\mathbf{u} \cdot \nabla) \mathbf{u}+\nabla \mathrm{p}=\mathbf{f}_{\mathrm{p}}(\mathbf{x}) \\
& \bullet \nabla \cdot \mathbf{u}=0
\end{aligned}
$$

subjected to the homogeneous boundary conditions

$$
\begin{aligned}
& \left.\bullet \mathbf{u}(\mathbf{x}, \mathrm{t})\right|_{\mathbf{x} \in \partial \mathrm{B}}=\lim _{|\mathbf{x}| \rightarrow \infty} \mathbf{u}(\mathbf{x}, \mathrm{t})=\mathbf{0} ; \\
& \text { - } \lim _{|\mathbf{x}| \rightarrow \infty} \mathrm{p}(\mathbf{x}, \mathrm{t})=0 .
\end{aligned}
$$

The discrete version of (2.2) will be addressed next.

\section{Boundary Conditions and "Wake Impedance"}

In order to deal with (2.2) one must specify, first of all, a finite fluid region R, as shown in Fig.(1), where the flow variables will be discretized by Finite Elements. Boundary conditions must be imposed on the border $\partial \mathrm{R}$ of $\mathrm{R}$ : only with them the "fluid flow operator" can be properly defined within R.

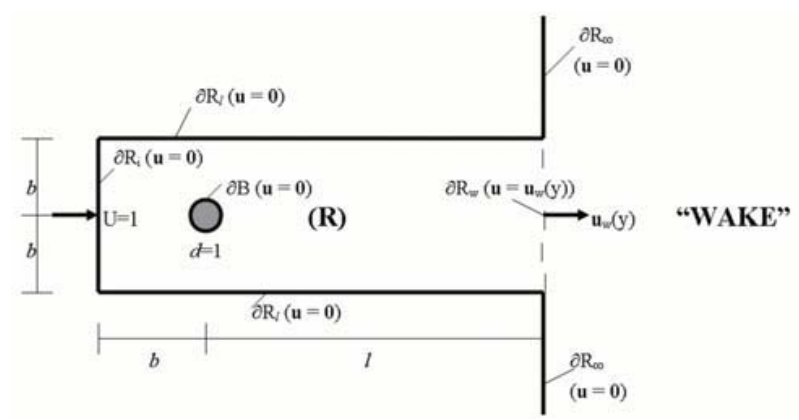

Figure 1. Finite fluid region $\mathbf{R}(\partial \mathbf{R}=\partial \mathbf{B} \cup \partial \mathbf{R} i \partial \mathbf{R} l \cup \partial \mathbf{R} w)$ and wake.

$$
\left(\mathbf{u}_{\mathrm{T}}(\mathbf{x}, \mathrm{t})=\mathbf{u}_{\mathrm{p}}(\mathbf{x})+\mathbf{u}(\mathbf{x}, \mathrm{t}) ; \mathbf{u}_{\mathrm{p}}(\mathbf{x}) \cong \mathbf{i} \text { for }|\mathbf{x}| \geq b\right) .
$$

The boundary $\partial \mathrm{R}$ is made by the cross section contour line $\partial \mathrm{B}$, by the "inlet" $\partial \mathrm{R}_{\mathrm{i}}$ at the vertical line $\mathrm{x}=-b$, by the "lateral sides" $\partial \mathrm{R}_{l}$ at $\mathrm{y}= \pm b$ and by the "outlet" $\partial \mathrm{R}_{\mathrm{w}}$ at $\mathrm{x}=l$ that defines the interface between $\mathrm{R}$ and the wake region $\mathrm{x}>l(\partial \mathrm{R}=$ $\left.\partial \mathrm{B} \cup \partial \mathrm{R}_{\mathrm{i}} \cup \partial \mathrm{R}_{l} \cup \partial \mathrm{R}_{\mathrm{w}}\right)$. The velocity field $\mathbf{u}(\mathbf{x}, \mathbf{t})$ is certainly null at $\partial \mathrm{B}$ and if $b$ is large enough $(b \approx 5 d)$ and $l$ is not much larger than $b(l \approx$ $10 d)$ it seems reasonable to assume that $\mathbf{u}(\mathbf{x}, \mathbf{t})$ is also null at $\partial \mathrm{R}_{\mathrm{i}} \cup \partial \mathrm{R}_{l}$ : the presence of the cylinder should not perturb the incoming flow at sufficient distance both upwind and laterally. The following essential boundary condition is thus assumed on this part of $\partial \mathrm{R}$ :

$$
\left.\mathbf{u}(\mathbf{x}, \mathrm{t})\right|_{\mathbf{x} \in \partial \mathrm{B} \cup \partial \mathrm{R}_{\mathrm{i}} \cup \partial \mathrm{R}_{l}}=\mathbf{0} .
$$

The same homogeneous boundary condition cannot be extended to the "outlet" $\partial \mathrm{R}_{\mathrm{w}}$ unless the distance $l$ is very large (and so it must be $b$ ). In fact, the vorticity generated at the cylinder dies out very slowly downstream, typically in a distance of order $l \approx \operatorname{Re}$ for the largest wavelength, and since $b$ increases roughly with $l^{1 / 2}$ the 
condition (2.3a) could be pushed to $\partial \mathrm{R}_{\mathrm{w}}$ only if the finite region $\mathrm{R}$ becomes very large ${ }^{2}$.

The velocity field at $\partial \mathrm{R}_{\mathrm{w}}$ should thus remain unspecified while the proper boundary condition at the outlet of $\mathrm{R}$ will be defined below in this section. If now the dynamic equation in $(2.2 \mathrm{~b})$ is multiplied by a virtual velocity $\delta \mathbf{u}(\mathbf{x})$ that satisfies, as usual, the same essential boundary condition (2.3a), and the continuity equation in (2.2b) is multiplied by $\delta \mathrm{p}(\mathbf{x})$ and both expressions are further integrated in $\mathrm{R}$ one obtains, after partial integration, that

$\cdot \int_{\mathrm{R}} \frac{\partial \mathbf{u}}{\partial \mathrm{t}} \cdot \delta \mathbf{u} \mathrm{dR}+\int_{\mathrm{R}}\left\{\left[\left(\mathbf{u}_{\mathrm{p}} \cdot \nabla\right) \mathbf{u}+(\mathbf{u} \cdot \nabla) \mathbf{u}_{\mathrm{p}}\right] \cdot \delta \mathbf{u}+\frac{1}{\mathrm{Re}}(\nabla \mathrm{u} \cdot \nabla(\delta \mathrm{u})+\nabla \mathrm{v} \cdot \nabla(\delta \mathrm{v}))\right\} \mathrm{dR}+$

$+\int_{\mathrm{R}}(\mathbf{u} \cdot \nabla) \mathbf{u} \cdot \delta \mathbf{u} \mathrm{dR}-\int_{\mathrm{R}} \mathrm{p}(\nabla \cdot \delta \mathbf{u}) \mathrm{dR}=\int_{\mathrm{R}} \mathbf{f}_{\mathrm{p}}(\mathbf{x}) \cdot \delta \mathbf{u} \mathrm{dR}+\mathrm{I}\left(\mathbf{u}_{\mathrm{w}}(\mathrm{y}) ; \delta \mathbf{u}_{\mathrm{w}}(\mathrm{y})\right) ;$

$\cdot \int \delta \mathrm{p}(\nabla \cdot \mathbf{u}) \mathrm{dR}=0$.

In (2.3b) the notation $\left\{\left(\mathbf{u}_{\mathrm{w}}(\mathrm{y}, \mathrm{t}) ; \delta \mathbf{u}_{\mathrm{w}}(\mathrm{y})\right) \equiv(\mathbf{u}(l, \mathrm{y}, \mathrm{t}) ; \delta \mathbf{u}(l, \mathrm{y})) ;|\mathrm{y}| \leq\right.$ $b$ \} was used to define the velocity and the virtual velocity over $\partial \mathrm{R}_{\mathrm{w}}$ and $\mathrm{I}\left(\mathbf{u}_{\mathrm{w}}(\mathrm{y}) ; \delta \mathbf{u}_{\mathrm{w}}(\mathrm{y})\right)$ is the "wake impedance", namely,

$\mathrm{I}\left(\mathbf{u}_{\mathrm{w}}(\mathrm{y}) ; \delta \mathbf{u}_{\mathrm{w}}(\mathrm{y})\right)=\int_{-b}^{b}\left[\left(-\mathrm{p}+\frac{1}{\operatorname{Re}} \frac{\partial \mathrm{u}}{\partial \mathrm{x}}\right)_{\mathrm{w}} \cdot \delta \mathrm{u}_{\mathrm{w}}(\mathrm{y})+\left(\frac{1}{\operatorname{Re}} \frac{\partial \mathrm{v}}{\partial \mathrm{x}}\right)_{\mathrm{w}} \cdot \delta \mathrm{v}_{\mathrm{w}}(\mathrm{y})\right] \mathrm{dy}$

where, assuming continuity, $(\cdot)_{\mathrm{w}}$ stands for the stress field in the wake at $\mathrm{x}=l$ : the "wake impedance" is thus the virtual power done by this stress field on the virtual velocity $\delta \mathbf{u}_{\mathrm{w}}(\mathrm{y})$ and it represents the "resistance" offered by the wake for the flow within the finite fluid region $\mathrm{R}$.

The wake region is bounded on the left by $\partial \mathrm{R}_{\mathrm{w}}$ and by two semiinfinite lines $\partial \mathbf{R}_{\infty}$, as indicated in Fig.(1). Since $\mathbf{u}(\mathbf{x}, \mathbf{t})$ was assumed null at the lateral sides $\partial \mathrm{R}_{l}$ it is certainly consistent with this assumption to take $\mathbf{u}(\mathbf{x}, \mathbf{t}) \equiv \mathbf{0}$ on $\partial \mathbf{R}_{\infty}$, since the perturbation caused by the cylinder should be even smaller over $\partial \mathrm{R}_{\infty}$ than over $\partial \mathrm{R}_{l}$. It turns out then that the flow in the wake is forced solely by the field $\mathbf{u}_{\mathrm{w}}(\mathrm{y}, \mathrm{t})$ and thus

$$
\begin{aligned}
& \left(-\mathrm{p}+\frac{1}{\operatorname{Re}} \frac{\partial \mathrm{u}}{\partial \mathrm{x}}\right)_{\mathrm{w}}=\mathrm{F}_{\mathrm{X}}\left(\mathbf{u}_{\mathrm{w}}\right) ; \\
& \left(\frac{1}{\operatorname{Re}} \frac{\partial \mathrm{v}}{\partial \mathrm{x}}\right)_{\mathrm{w}}=\mathrm{F}_{\mathrm{Y}}\left(\mathbf{u}_{\mathrm{w}}\right),
\end{aligned}
$$

where the functionals $\Phi_{\mathrm{X}, \mathrm{Y}}(\cdot)$ can be determined by solving the flow problem in the wake. If both the velocity and virtual velocity $\left\{\mathbf{u}_{\mathrm{w}}(\mathrm{y}, \mathrm{t}) ; \delta \mathbf{u}_{\mathrm{w}}(\mathrm{y})\right\}$ at the outlet are discretized as

$$
\left\{\begin{array}{c}
\mathbf{u}_{\mathrm{w}}(\mathrm{y}, \mathrm{t}) \\
\delta \mathbf{u}_{\mathrm{w}}(\mathrm{y})
\end{array}\right\}=\sum_{\mathrm{k}=1}^{\mathrm{N}_{\mathrm{w}}}\left(\left\{\begin{array}{c}
\mathrm{U}_{\mathrm{w}, \mathrm{k}}(\mathrm{t}) \\
\delta \mathrm{U}_{\mathrm{w}, \mathrm{k}}
\end{array}\right\} \mathbf{i}+\left\{\begin{array}{c}
\mathrm{U}_{\mathrm{w}, \mathrm{k}+\mathrm{N}_{\mathrm{w}}}(\mathrm{t}) \\
\delta \mathrm{U}_{\mathrm{w}, \mathrm{k}+\mathrm{N}_{\mathrm{w}}}
\end{array}\right\} \mathbf{j}\right) \cdot \mathrm{h}_{\mathrm{k}, \mathrm{w}}(\mathrm{y}),
$$

where $\left\{\mathrm{h}_{\mathrm{k}, \mathrm{w}}(\mathrm{y}) ; \mathrm{k}=1,2, \ldots, \mathrm{N}_{\mathrm{w}}\right\}$ are the interpolation functions for the velocity field restricted to $\partial \mathrm{R}_{\mathrm{w}}$ and $\left\{\mathbf{U}_{\mathrm{w}}(\mathrm{t}) ; \delta \mathbf{U}_{\mathrm{w}}\right\}$ are the nodal values vector, it can be shown that

2 This seems to be true even for a "small" Reynolds number: for $\mathrm{Re}=41$ the wake has already begun to oscillate sinusoidally far downstream, see Van Dyke (1982), plate 46. The existing numerical results predict, as a rule, a critical Reynolds number above 40 (Recr $\approx 45$ ) although the expected value should be below $(\operatorname{Recr} \approx 35)$.

\section{J. of the Braz. Soc. of Mech. Sci. \& Eng. Copyright $(2004$ by ABCM}

$$
\begin{aligned}
& \bullet \mathbf{I}\left(\mathbf{u}_{\mathrm{w}}(\mathrm{y}) ; \delta \mathbf{u}_{\mathrm{w}}(\mathrm{y})\right)=\delta \mathbf{U}_{\mathrm{w}}^{\mathrm{t}} \cdot \mathbf{I}\left(\mathbf{U}_{\mathrm{w}}\right) \\
& \bullet \mathbf{I}\left(\mathbf{U}_{\mathrm{w}}\right)=-\left[\mathbf{M}_{\mathrm{w}} \cdot \dot{\mathbf{U}}_{\mathrm{w}}+\mathbf{K}_{\mathrm{w}} \cdot \mathbf{U}_{\mathrm{w}}+\mathbf{N}_{\mathrm{w}}\left(\mathbf{U}_{\mathrm{w}}\right) \cdot \mathbf{U}_{\mathrm{w}}\right],
\end{aligned}
$$

the matrices $\left\{\mathbf{M}_{\mathrm{w}} ; \mathbf{K}_{\mathrm{w}} ; \mathbf{N}_{\mathrm{w}}\left(\mathbf{U}_{\mathrm{w}}\right)\right\}$ being computed from explicitly defined Fourier series ${ }^{3}$.

Within $\mathrm{R}$ the discrete velocity and pressure fields can be expressed as

$$
\begin{aligned}
& \mathbf{u}(\mathbf{x}, \mathrm{t})=\sum_{\mathrm{k}=1}^{\mathrm{N}}\left(\mathrm{U}_{\mathrm{k}}(\mathrm{t}) \mathbf{i}+\mathrm{U}_{\mathrm{k}+\mathrm{N}}(\mathrm{t}) \mathbf{j}\right) \cdot \mathrm{h}_{\mathrm{k}}(\mathbf{x}) ; \\
& \mathrm{p}(\mathbf{x}, \mathrm{t})=\sum_{\alpha=1}^{\mathrm{e}} \mathrm{P}_{\alpha}(\mathrm{t}) \cdot \mathrm{t}_{\alpha}(\mathbf{x}),
\end{aligned}
$$

with $\left\{\mathrm{h}_{\mathrm{k}}(\mathbf{x}) ; \mathrm{k}=1,2, \ldots, \mathrm{N}\right\}$ and $\left\{\mathrm{t}_{\alpha}(\mathbf{x}) ; \alpha=1,2, \ldots, \mathrm{e}\right\}$ being, respectively, the interpolating functions for the velocity and pressure fields. The functions $\left\{h_{k}(\mathbf{x})\right\}$ are necessarily continuous but the $\left\{t_{\alpha}(x)\right\}$ may or may not be so; in reality, the pressure field does work on $\nabla \cdot \delta \mathbf{u}$ and it seems reasonable to choose the $\left\{\mathrm{t}_{\alpha}(\mathbf{x})\right\}$ in conformity with the discrete field $\nabla \cdot \delta \mathbf{u}$ obtained from the $\left\{\mathrm{h}_{\mathrm{k}}(\mathbf{x})\right\}$. Placing (2.5a) into the integrals that appear in (2.3b) and defining the matrices

$\int_{\mathrm{R}}^{\partial} \frac{\partial \mathbf{u}}{\partial t} \cdot \delta \mathbf{u d R}=\delta \mathbf{U}^{\mathrm{t}} \cdot \mathbf{M}_{\mathrm{R}} \cdot \dot{\mathbf{U}} ; \int_{\mathrm{R}}(\mathbf{u} \cdot \nabla) \mathbf{u} \cdot \delta \mathbf{u} \mathrm{dR}=\delta \mathbf{U}^{\mathrm{t}} \cdot \mathbf{N}_{\mathrm{R}}(\mathbf{U}) \cdot \mathbf{U} ;$

$\int_{\mathrm{R}}\left[\left(\mathbf{u}_{\mathrm{p}} \cdot \nabla\right) \mathbf{u}+(\mathbf{u} \cdot \nabla) \mathbf{u}_{\mathrm{p}}\right] \cdot \delta \mathbf{u} \mathrm{dR}+\frac{1}{\mathrm{Re}_{\mathrm{R}}} \int_{\mathrm{R}}(\nabla \mathrm{u} \cdot \nabla(\delta \mathrm{u})+\nabla \mathrm{v} \cdot \nabla(\delta \mathrm{v})) \mathrm{dR}=\delta \mathbf{U}^{\mathrm{t}} \cdot \mathbf{K}_{\mathrm{p}, \mathrm{R}} \cdot \mathbf{U} ;$ $\int_{\mathrm{R}} \mathrm{p}(\nabla \cdot \delta \mathbf{u}) \mathrm{dR}=\delta \mathbf{U}^{\mathrm{t}} \cdot \mathbf{R} \cdot \mathbf{P} ; \int_{\mathrm{R}} \delta \mathrm{p}(\nabla \cdot \mathbf{u}) \mathrm{dR}=\delta \mathbf{P}^{\mathrm{t}} \cdot \mathbf{R}^{\mathrm{t}} \cdot \mathbf{U} ;$

$\int_{\mathrm{R}} \mathbf{f}_{\mathrm{p}}(\mathbf{x}) \cdot \delta \mathbf{u} \mathrm{dR}=\delta \mathbf{U}^{\mathrm{t}} \cdot \mathbf{F}_{\mathrm{p}}$,

the discrete form of the flow equation in weak form reads (see $(2.3 b)$ and $(2.4 c))$

$\bullet \delta \mathbf{U}^{\mathrm{t}} \cdot\left\{\mathbf{M}_{\mathrm{R}} \cdot \dot{\mathbf{U}}+\mathbf{K}_{\mathrm{p}, \mathrm{R}} \cdot \mathbf{U}+\mathbf{N}_{\mathrm{R}}(\mathbf{U}) \cdot \mathbf{U}-\mathbf{R} \cdot \mathbf{P}\right\}=$
$=-\delta \mathbf{U}_{\mathrm{w}}^{\mathrm{t}} \cdot\left\{\mathbf{M}_{\mathrm{w}} \cdot \dot{\mathbf{U}}_{\mathrm{w}}+\mathbf{K}_{\mathrm{w}} \cdot \mathbf{U}_{\mathrm{w}}+\mathbf{N}_{\mathrm{w}}\left(\mathbf{U}_{\mathrm{w}}\right) \cdot \mathbf{U}_{\mathrm{w}}\right\}+\delta \mathbf{U}^{\mathrm{t}} \cdot \mathbf{F}_{\mathrm{p}}$

$\bullet \mathbf{R}^{\mathrm{t}} \cdot \mathbf{U}=\mathbf{0}$.

Observing that $\left\{\mathbf{U}_{\mathrm{w}} ; \delta \mathbf{U}_{\mathrm{w}}\right\}$ are, in fact, the part of $\{\mathbf{U} ; \delta \mathbf{U}\}$ defined in $\partial \mathrm{R}_{\mathrm{w}}$, one can take the "wake impedance" on the left side of $(2.5 \mathrm{c})$ and sum both contributions to obtain

$$
\begin{aligned}
& \bullet \delta \mathbf{U}^{\mathrm{t}} \cdot\left\{\mathbf{M} \cdot \dot{\mathbf{U}}+\mathbf{K}_{\mathrm{p}} \cdot \mathbf{U}+\mathbf{N}(\mathbf{U}) \cdot \mathbf{U}-\mathbf{R} \cdot \mathbf{P}\right\}=\delta \mathbf{U}^{\mathrm{t}} \cdot \mathbf{F}_{\mathrm{p}} \\
& \bullet \mathbf{R}^{\mathrm{t}} \cdot \mathbf{U}=\mathbf{0}
\end{aligned}
$$

The dynamic parcels of (2.6), proportional to $\left\{\mathbf{M} ; \mathbf{K}_{\mathrm{p}} ; \mathbf{N}(\mathbf{U})\right\}$, come both from the finite fluid region $\mathrm{R}$, where the flow variables are discretized, and the wake region downstream: $\mathbf{M}$ is the local inertia matrix, $\mathbf{K}_{\mathrm{p}}$ represents, as indicated in $(2.5 \mathrm{~b})$, the influence of the viscous stress and the convective acceleration due to the auxiliary field $\mathbf{u}_{\mathrm{p}}(\mathbf{x})$ and $\mathbf{N}(\mathbf{U}) \cdot \mathbf{U}$ is the convective inertia force. The parcels $\left\{\mathbf{R}^{\mathrm{t}} \cdot \mathbf{U} ; \mathbf{R} \cdot \mathbf{P}\right\}$ are due to mass conservation and the related constraint force (pressure) defined in the finite region R: the matrix

3 For the Fourier series expansion one must impose a finite breadth $2 \mathrm{~W}$ for the wake, with $2 \mathrm{~W}$ being arbitrarily large $(2 \mathrm{~W}>>\mathrm{b})$. It can be shown that the number of terms $\mathrm{nL}$ in these series increases both with $\mathrm{W}$ and $\mathrm{Re}$; typically, $\mathrm{nL}$ $\cong \mathrm{O}(\mathrm{W} \cdot(\mathrm{Re}) 1 / 2)$. 
- $\mathbf{R}$ represents the (discrete) gradient operator and $\mathbf{R}^{\mathrm{t}}$ the (discrete) divergence operator. Obviously, mass is already conserved in the "wake solution" (2.4c) and this constraint should not appear again at (2.6).

The (discrete) Navier-Stokes equation (2.6) emulates thus the structure of the original equation $(2.2 \mathrm{~b})$ and it will be analyzed next: in this discrete form the mathematical analysis is much simpler, once is based only on some general results in Linear Algebra, and, furthermore, its outcome has an operational appeal given its direct link to the final numerical results.

\section{The Solenoidal and Gradient Sub-Spaces}

Let $\mathrm{W}_{\mathrm{N}}$ be the $2 \mathrm{~N}$-dimensional linear space of the discrete velocity vector $\mathbf{U}$ and $\mathrm{L}_{\mathrm{e}}$ be the e-dimensional linear space of the discrete pressure vector $\mathbf{P}$; in both spaces it will be assumed the standard inner product $\langle\mathbf{U} ; \mathbf{V}\rangle=\mathbf{U}^{\mathrm{t}} \cdot \mathbf{V}$ and the related norm $\|\mathbf{U}\|^{2}=$ $\langle\mathbf{U} ; \mathbf{U}\rangle=\mathbf{U}^{\mathrm{t}} \cdot \mathbf{U}$.

Let also $\left\{\mathrm{J}_{\mathrm{s}} ; \mathrm{G}_{\mathrm{r}}\right\}$ be the s-dimensional and r-dimensional subspaces of $\mathrm{W}_{\mathrm{N}}$ defined by the relations

$$
\begin{aligned}
& \mathrm{J}_{\mathrm{s}}=\left\{\mathbf{V} \in \mathrm{W}_{\mathrm{N}}: \mathbf{R}^{\mathrm{t}} \cdot \mathbf{V}=\mathbf{0} \in \mathrm{L}_{\mathrm{e}}\right\} ; \\
& \mathrm{G}_{\mathrm{r}}=\left\{\mathbf{V} \in \mathrm{W}_{\mathrm{N}}: \mathbf{V}=\mathbf{R} \cdot \boldsymbol{\varphi} ; \boldsymbol{\varphi} \in \mathrm{L}_{\mathrm{e}}\right\} .
\end{aligned}
$$

Elements of $\mathrm{G}_{\mathrm{r}}$ are "gradients" of "scalar fields" $\varphi \in \mathrm{L}_{\mathrm{e}}$ and for this reason $\mathrm{G}_{\mathrm{r}}$ is called the "gradient sub-space" of $\mathrm{W}_{\mathrm{N}}$; notice that $\mathrm{G}_{\mathrm{r}}$ is generated by the linear combinations of the column vectors of R. Elements of $J_{\mathrm{s}}$ have null divergence and so $\mathrm{J}_{\mathrm{s}}$ is called the "solenoidal sub-space"; by definition, their elements are orthogonal to the column vectors of $\mathbf{R}$ and thus $\mathrm{J}_{\mathrm{s}}$ is the orthogonal complement of $\mathrm{G}_{\mathrm{r}}$ or $(\mathrm{r}+\mathrm{s}=2 \mathrm{~N})$

$$
\mathrm{W}_{\mathrm{N}}=\mathrm{J}_{\mathrm{s}} \oplus \mathrm{G}_{\mathrm{r}},
$$

see Ladyzenskaja (1969)

One introduces here the operators

$$
\begin{aligned}
& \bullet \boldsymbol{\Delta}=\mathbf{R}^{\mathrm{t}} \cdot \mathbf{R}: \mathrm{L}_{\mathrm{e}} \rightarrow \mathrm{L}_{\mathrm{e}} ; \\
& \bullet \nabla=\mathbf{R} \cdot \mathbf{R}^{\mathrm{t}}: \mathrm{W}_{\mathrm{N}} \rightarrow \mathrm{W}_{\mathrm{N}},
\end{aligned}
$$

where $\Delta$ is the (discrete) Laplacian operator and $\nabla$ will be named the "conjugated Laplacian". Both $\Delta$ and $\nabla$ are represented by symmetric, positive semi-definite sparse matrices, the "sparseness" being a consequence of the "local character" of the Finite Element discretization.

By definition $\mathbf{R}^{\mathrm{t}} \cdot \mathbf{V}=\mathbf{0}$ if $\mathbf{V} \in \mathrm{J}_{\mathrm{s}}$ and so $\nabla \cdot \mathbf{V}=\mathbf{0}$ or $\mathrm{J}_{\mathrm{s}} \subseteq$ Null $(\nabla)$. In reality, it can be shown that $\mathrm{J}_{\mathrm{s}} \equiv$ Null $(\nabla)$. Let $\left\{\mathbf{T}_{\alpha} ; \alpha=\right.$ $1,2, \ldots, \mathrm{s}\}$ be an othonormal basis of Null $(\nabla) \equiv \mathbf{J}_{\mathrm{s}}$ and $\left\{\mathbf{G}_{\mathrm{j}} ; \mathrm{j}=\right.$ $1,2, \ldots, \mathrm{r}\}$ be the orthonormal eigenvectors corresponding to the positive spectrum $\left\{\kappa_{\mathrm{j}}>0 ; \mathrm{j}=1,2, \ldots, \mathrm{r}\right\}$ of $\nabla$, namely:

$$
\begin{aligned}
& \bullet \nabla \cdot \mathbf{T}_{\alpha}=\mathbf{0} ; \alpha=1,2, \ldots, \mathrm{s} ; \\
& \bullet \nabla \cdot \mathbf{G}_{\mathrm{j}}=\kappa_{\mathrm{j}} \mathbf{G}_{\mathrm{j}} ; \mathrm{j}=1,2, \ldots, \mathrm{r} .
\end{aligned}
$$

Obviously $\left\{\mathbf{G}_{1} ; \mathbf{G}_{2} ; \ldots ; \mathbf{G}_{\mathrm{r}}\right\}$ is a basis of $\mathrm{G}_{\mathrm{r}}$ while $\left\{\mathbf{T}_{1} ; \mathbf{T}_{2}\right.$; $\left.\ldots ; \mathbf{T}_{\mathrm{s}}\right\}$ is a basis of $\mathrm{J}_{\mathrm{s}}$; assuming that $0<\kappa_{1} \leq \kappa_{2} \leq \ldots \leq \kappa_{\mathrm{r}}$ consider the matrix

$$
\nabla_{1}=\mathbf{I}-\frac{1}{\kappa_{\mathrm{r}}} \nabla
$$

Certainly $\nabla_{1}$ is a symmetric, positive semi-definite sparse matrix with a spectrum in the interval $[0 ; 1]$; furthermore

$$
\bullet \nabla_{1} \cdot \mathbf{T}_{\alpha}=\mathbf{T}_{\alpha},
$$

a relation that can be used to determine an orthonormal basis of the solenoidal sub-space ${ }^{4}$.

The Laplacian operator $\Delta$ may have a non-empty null sub-space $(\operatorname{Null}(\Delta) \neq \varnothing)$ but it certainly has a positive spectrum; in fact, if

$$
\bullet \hat{\mathbf{G}}_{\mathrm{j}}=\frac{1}{\sqrt{\kappa_{\mathrm{j}}}} \mathbf{R}^{\mathrm{t}} \cdot \mathbf{G}_{\mathrm{j}}
$$

then one can easily check that $\left\|\hat{\mathbf{G}}_{\mathrm{j}}\right\|=1$ with

$$
\bullet \boldsymbol{\Delta} \cdot \hat{\mathbf{G}}_{\mathrm{j}}=\kappa_{\mathrm{j}} \hat{\mathbf{G}}_{\mathrm{j}} .
$$

Observing now the conjugated relation

$$
\bullet \mathbf{G}_{\mathrm{j}}=\frac{1}{\sqrt{\kappa_{\mathrm{j}}}} \mathbf{R} \cdot \hat{\mathbf{G}}_{\mathrm{j}}
$$

the following result can be derived: the positive spectrum of $\Delta$ must coincide, necessarily, with the positive spectrum $\left\{\kappa_{\mathrm{j}}>0 ; \mathrm{j}=\right.$ $1,2, \ldots, \mathrm{r}\}$ of $\nabla$. In fact, if $\kappa \neq \kappa_{\mathrm{j}}$ were a positive eigenvalue of $\Delta$ with eigenvector $\hat{\mathbf{G}}$ then $\mathbf{G}=\mathbf{R} \cdot \hat{\mathbf{G}}$ should be an eigenvector of $\nabla$ with the same eigenvalue $\kappa$ and so $\kappa \in\left\{\kappa_{j}>0 ; j=1,2, \ldots, r\right\}$ once, by definition, this is the set of all positive eigenvalues of $\nabla$. The operators $\{\Delta ; \nabla\}$ establish, thus, a duality between the sub-space $\hat{\mathrm{G}}_{\mathrm{r}} \subset \mathrm{L}_{\mathrm{e}}$, generated by the vectors $\left\{\hat{\mathbf{G}}_{1} ; \hat{\mathbf{G}}_{2} ; \ldots ; \hat{\mathbf{G}}_{\mathrm{r}}\right\}$, and the gradient sub-space $\mathrm{G}_{\mathrm{r}} \subset \mathrm{W}_{\mathrm{N}}$ : if $\varphi \in \hat{\mathrm{G}}_{\mathrm{r}}$ then $\mathbf{R} \cdot \varphi \in \mathrm{G}_{\mathrm{r}}$ and if $\mathbf{V} \in \mathrm{G}_{\mathrm{r}}$ then $\mathbf{R}^{\mathrm{t}} \cdot \mathbf{V} \in \hat{\mathrm{G}}_{\mathrm{r}}$; the sub-space Null $(\Delta)$ is the orthogonal complement of $\hat{\mathrm{G}}_{\mathrm{r}}$ and so

$$
\begin{aligned}
& \mathrm{L}_{\mathrm{e}}=\operatorname{Null}(\boldsymbol{\Delta}) \oplus \hat{\mathrm{G}}_{\mathrm{r}} ; \\
& \mathrm{W}_{\mathrm{N}}=\operatorname{Null}(\nabla) \oplus \mathrm{G}_{\mathrm{r}} .
\end{aligned}
$$

Let $\left\{\mathbf{S}_{\alpha} ; \alpha=1,2, \ldots, \mathrm{e}-\mathrm{r}\right\}$ be an orthonormal basis of Null $(\Delta)$, named the "spurious pressure modes" in the specialized literature, see Gunzburger (1985); they satisfy the relations

$$
\bullet \boldsymbol{\Delta} \cdot \mathbf{S}_{\alpha}=\mathbf{0} ; \alpha=1,2, \ldots, \mathrm{e}-\mathrm{r}
$$

and, as it will be seen in the next item, these modes play in the discrete problem the same role played by the constant pressure field in the continuum problem, namely: they do not interfere with the dynamics of the flow. In accordance with (2.9b) one introduces here the matrix

$$
\bullet \boldsymbol{\Delta}_{1}=\mathbf{I}-\frac{1}{\kappa_{\mathrm{r}}} \boldsymbol{\Delta}
$$

where, again, $\Delta_{1}$ is a symmetric, positive semi-definite sparse matrix with spectrum in the interval $[0 ; 1]$; this matrix will be used in the next item in the context of the Poisson's equation for the (discrete) pressure field.

It seems worthwhile to finish this section with a more technical remark about the Finite Element discretization, related to the so called "div-stability condition" (Ladyzhenskaya - Babuska - Brezzi

4 The ARPACK algorithm is specially suited to deal with eigenvalue problems of a large sparse matrices, see Lehoucq \& Sorensen \& Yang(1997). 
condition). The point is that for some classes of Finite Elements (FE) the smallest eigenvalue $\kappa_{1}$ becomes "too small" as the mesh size $h$ goes to zero, indicating that elements of the gradient subspace $\mathrm{G}_{\mathrm{r}}$ tend to "slip" into the solenoidal sub-space $\mathrm{J}_{\mathrm{s}}$ as $h \rightarrow 0$. In this case the solenoidal sub-space $J_{\mathrm{s}}$ becomes "too rarefied" once at least some of the solenoidal fields are, in fact, "slipping modes" of $\mathrm{G}_{\mathrm{r}}$; this problem is particularly acute for the simplest FE discretization, where the velocity field is linear piecewise continuous and the pressure is constant in each element, see Gunzburger (1985) and Bathe (1996), for example. However, as shown in Aranha (2003), if the actual solenoidal sub-space is enlarged by these "slipping modes" in a way dictated by the "divstability condition" this problem can be overcome without impairing the standard Finite Element convergence rate. In the present context some of the eigenvalues-eigenmodes in $\mathrm{G}_{\mathrm{r}}$ are naturally computed in the effort to determine the basis $\left\{\mathbf{T}_{\alpha} ; \alpha=1,2, \ldots, \mathrm{s}\right\}$ of $\mathrm{J}_{\mathrm{s}}$ and this "enlarging" process can then be worked out easily; or, in short, questions related to the "div-stability condition" are of no special concern here.

\section{The Solenoidal Velocity Field and Poisson's Equation}

The solution of the (discrete) Navier-Stokes equation (2.6) will be dealt in two stages: first, the projection of (2.6) into $\mathrm{J}_{\mathrm{s}}$ will result in a standard nonlinear differential equation for the velocity; second, Poisson's equation for the pressure will be derived by projecting (2.6) into $\mathrm{G}_{\mathrm{r}}$. The simple structure of the dynamic equation in $\mathrm{J}_{\mathrm{s}}$ allows one to develop standard asymptotic analysis for the underlying nonlinear system and to obtain, in this way, Landau's equation in the vicinity of the Hopf bifurcation; in the other side, it is possible to show, with the help of item (2.2), that Poisson's equation has a solution and that this solution is "unique". With this purpose in mind one introduces the matrices

$$
\begin{aligned}
& \bullet \mathbf{T}=\left[\mathbf{T}_{1} ; \mathbf{T}_{2} ; \ldots ; \mathbf{T}_{\mathrm{s}}\right] \\
& \bullet \mathbf{M}_{\mathrm{s}}=\mathbf{T}^{\mathrm{t}} \cdot \mathbf{M} \cdot \mathbf{T} ; \\
& \bullet \mathbf{K}_{\mathrm{p}, \mathrm{s}}=\mathbf{T}^{\mathrm{t}} \cdot \mathbf{K}_{\mathrm{p}} \cdot \mathbf{T} ; \\
& \bullet \mathbf{N}_{\mathrm{s}}(\mathbf{q})=\mathbf{T}^{\mathrm{t}} \cdot \mathbf{N}(\mathbf{T} \cdot \mathbf{q}) \cdot \mathbf{T} ; \\
& \bullet \mathbf{F}_{\mathrm{p}, \mathrm{s}}=\mathbf{T}^{\mathrm{t}} \cdot \mathbf{F}_{\mathrm{p}},
\end{aligned}
$$

where $\mathbf{R}^{\mathrm{t}} \cdot \mathbf{T}=\mathbf{0}$ since $\mathbf{T}_{\alpha} \in \mathrm{J}_{\mathrm{s}}$; also, given arbitrary s-dimensional vectors $\{\mathbf{q}(\mathrm{t}) ; \delta \mathbf{q}\}$ one has

$$
\begin{aligned}
& \mathbf{U}(\mathrm{t}) \in \mathrm{J}_{\mathrm{s}} \Leftrightarrow \mathbf{U}(\mathrm{t})=\mathbf{T} \cdot \mathbf{q}(\mathrm{t}) ; \\
& \delta \mathbf{U} \in \mathrm{J}_{\mathrm{s}} \Leftrightarrow \delta \mathbf{U}=\mathbf{T} \cdot \delta \mathbf{q} .
\end{aligned}
$$

The virtual velocity $\delta \mathbf{U} \in \mathrm{W}_{\mathrm{N}}$ in (2.6) belongs either to $\mathrm{J}_{\mathrm{s}}$ or to $\mathrm{G}_{\mathrm{r}}$; assuming first $\delta \mathbf{U}=\mathbf{T} \cdot \delta \mathbf{q} \in \mathrm{J}_{\mathrm{s}}$ and recalling that $\mathbf{T}^{\mathrm{t}} \cdot \mathbf{R}=\left(\mathbf{R}^{\mathrm{t}} \cdot \mathbf{T}\right)^{\mathrm{t}}=$ $\mathbf{0}^{\mathrm{t}}$ one obtains, with the help of (2.12b), the following equation for the variable $\mathbf{q}(\mathrm{t})$ (see (2.12a)):

$$
\mathbf{M}_{\mathrm{s}} \cdot \dot{\mathbf{q}}+\mathbf{K}_{\mathrm{p}, \mathrm{s}} \cdot \mathbf{q}+\mathbf{N}_{\mathrm{s}}(\mathbf{q}) \cdot \mathbf{q}=\mathbf{F}_{\mathrm{p}, \mathrm{s}} \cdot
$$

The asymptotic solution of (2.13), leading to Landau's equation, will be addressed in item (2.5). Introducing the dynamic force vector (see (2.6))

$$
\mathbf{F}_{\mathrm{D}}(\mathrm{t})=\mathbf{M} \cdot \dot{\mathbf{U}}+\mathbf{K}_{\mathrm{p}} \cdot \mathbf{U}+\mathbf{N}(\mathbf{U}) \cdot \mathbf{U}-\mathbf{F}_{\mathrm{p}} \in \mathrm{W}_{\mathrm{N}},
$$

one can write $\Phi_{\mathrm{D}}(\mathrm{t})$ in the form $\left(\left\{\mathbf{T}_{\alpha}\right\} \cup\left\{\mathbf{G}_{\mathrm{j}}\right\}\right.$ is an orthonormal basis of $\mathrm{W}_{\mathrm{N}}$ )

$$
\begin{aligned}
& \mathbf{F}_{\mathrm{D}}(\mathrm{t})=\sum_{\alpha=1}^{\mathrm{s}} \mathrm{f}_{\mathrm{d}, \alpha}(\mathrm{t}) \cdot \mathbf{T}_{\alpha}+\sum_{\mathrm{j}=1}^{\mathrm{r}} \mathrm{f}_{\mathrm{d}, \mathrm{j}}(\mathrm{t}) \cdot \mathbf{G}_{\mathrm{j}} ; \\
& \mathrm{f}_{\mathrm{d}, \alpha}(\mathrm{t})=\left\langle\mathbf{F}_{\mathrm{D}}(\mathrm{t}) ; \mathbf{T}_{\alpha}\right\rangle ; \mathrm{f}_{\mathrm{d}, \mathrm{j}}(\mathrm{t})=\left\langle\mathbf{F}_{\mathrm{D}}(\mathrm{t}) ; \mathbf{G}_{\mathrm{j}}\right\rangle .
\end{aligned}
$$

By placing now $\delta \mathbf{U}=\mathbf{R} \cdot \delta \varphi \in \mathrm{G}_{\mathrm{r}}$ in (2.6) one obtains, with the help of (2.8) and (2.10a), the following Poisson's equation for the pressure $\mathbf{P}$

$$
\bullet \boldsymbol{\Delta} \cdot \mathbf{P}=\mathbf{R}^{\mathrm{t}} \cdot \mathbf{F}_{\mathrm{D}}(\mathrm{t})=\sum_{\mathrm{j}=1}^{\mathrm{r}} \mathrm{f}_{\mathrm{d}, \mathrm{j}}(\mathrm{t}) \sqrt{\kappa_{\mathrm{j}}} \cdot \hat{\mathbf{G}}_{\mathrm{j}} \in \hat{\mathrm{G}}_{\mathrm{r}}
$$

since $\mathbf{R}^{\mathrm{t}} \cdot \mathbf{T}_{\alpha} \equiv \mathbf{0}\left(\mathbf{T}_{\alpha} \in \mathrm{J}_{\mathrm{s}}\right)$. But $\mathbf{P} \in \mathrm{L}_{\mathrm{e}}$ and $\left\{\mathbf{S}_{\alpha} ; \alpha=1,2, . ., \mathrm{e}-\right.$ $\mathbf{r}\} \cup\left\{\hat{\mathbf{G}}_{\mathbf{j}} ; \mathrm{j}=1,2, \ldots, \mathbf{r}\right\}$ is an orthonormal basis of $\mathrm{L}_{\mathrm{e}}$ : expressing $\mathbf{P}$ in this basis and using (2.10b) it is easy to check that the general solution of (2.14c) is given by (recall that $\Delta \cdot \mathbf{S}_{\alpha}=\mathbf{0}$ )

$$
\mathbf{P}=\underbrace{\sum_{\alpha=1}^{\mathrm{e}-\mathrm{r}} a_{\alpha} \cdot \mathbf{S}_{\alpha}}_{\mathbf{P}_{\mathrm{s}}}+\sum_{\mathrm{j}=1}^{\mathrm{r}} \frac{\mathrm{f}_{\mathrm{d}, \mathrm{j}}(\mathrm{t})}{\sqrt{\kappa_{\mathrm{j}}}} \cdot \hat{\mathbf{G}}_{\mathrm{j}},
$$

where $\left\{a_{\alpha} ; \alpha=1,2, \ldots, \mathrm{e}-\mathrm{r}\right\}$ are arbitrary coefficients. The "spurious mode" $\mathbf{P}_{\mathrm{s}}=\Sigma a_{\alpha} \cdot \mathbf{S}_{\alpha}$ plays here the same role played by the constant pressure field in the continuum problem: in fact, its (discrete) gradient $\mathbf{R} \cdot \mathbf{P}_{\mathrm{s}}$ - and it is in this way that the pressure appears in the discrete flow equation (2.6) - is null since $\Delta \cdot \mathbf{P}_{\mathrm{s}} \equiv \mathbf{0}$ (recall that $\Delta \cdot \mathbf{S}_{\alpha}$ $=\mathbf{0})$ and

$$
\left\|\mathbf{R} \cdot \mathbf{P}_{\mathrm{s}}\right\|^{2}=\mathbf{P}_{\mathrm{s}}^{\mathrm{t}} \cdot \mathbf{R}^{\mathrm{t}} \cdot \mathbf{R} \cdot \mathbf{P}_{\mathrm{s}}=\mathbf{P}_{\mathrm{s}}^{\mathrm{t}} \cdot \Delta \cdot \mathbf{P}_{\mathrm{s}} \equiv 0
$$

The solution $\mathbf{P}$ of the Poisson's equation is thus "uniquely" defined by the expression

$$
\bullet P(t)=\sum_{j=1}^{r} \frac{f_{d, j}(t)}{\sqrt{\kappa_{j}}} \cdot \hat{\mathbf{G}}_{j},
$$

and it is important to point out that $\mathbf{P}(\mathrm{t})$ can be also obtained by repeated multiplications of the sparse matrix $\Delta_{1}$; in fact, from (2.11c) it follows that

$$
\Delta_{1} \cdot \hat{\mathbf{G}}_{\mathrm{j}}=\varepsilon_{\mathrm{j}} \hat{\mathbf{G}}_{\mathrm{j}} ; \varepsilon_{\mathrm{j}}=1-\kappa_{\mathrm{j}} / \kappa_{\mathrm{r}},
$$

and from the convergence of the geometric series $\Sigma\left(\varepsilon_{\mathrm{j}}\right)^{\mathrm{n}}=1 /\left(1-\varepsilon_{\mathrm{j}}\right)$ one obtains

$$
\bullet \mathbf{P}(\mathrm{t})=\frac{1}{\kappa_{\mathrm{r}}} \sum_{\mathrm{n}=0}^{\infty} \boldsymbol{\Delta}_{1}^{\mathrm{n}} \cdot \mathbf{R}^{\mathrm{t}} \cdot \mathbf{F}_{\mathrm{D}}(\mathrm{t})
$$

The series (2.15c) has an "almost geometric structure" and its convergence can be thus accelerated by Shanks Transformation, see Bender \& Orszag (1978).

\section{The Steady State Solution (Ue)}

Let $\mathbf{q}_{\mathrm{e}, \mathrm{p}}$ be the steady solution of (2.13), namely

$$
\bullet \mathbf{K}_{\mathrm{p}, \mathrm{s}} \cdot \mathbf{q}_{\mathrm{e}, \mathrm{p}}+\mathbf{N}_{\mathrm{s}}\left(\mathbf{q}_{\mathrm{e}, \mathrm{p}}\right) \cdot \mathbf{q}_{\mathrm{e}, \mathrm{p}}=\mathbf{F}_{\mathrm{p}, \mathrm{s}}
$$

where both $\mathbf{K}_{\mathrm{p}, \mathrm{s}}$ and $\mathbf{F}_{\mathrm{p}, \mathrm{s}}$ are functions of the Reynolds number ( $\mathbf{K}_{\mathrm{p}, \mathrm{s}}$ $\left.=\mathbf{K}_{\mathrm{p}, \mathrm{s}}(\operatorname{Re}) ; \mathbf{F}_{\mathrm{p}, \mathrm{s}}=\mathbf{F}_{\mathrm{p}, \mathrm{s}}(\mathrm{Re})\right)$ and so it is the steady solution: $\mathbf{q}_{\mathrm{e}, \mathrm{p}}=$ 
$\mathbf{q}_{\mathrm{e}, \mathrm{p}}(\operatorname{Re})$. If (2.16a) is differentiated with respect to Re and the matrix $\mathbf{K}_{\mathrm{s}}=\mathbf{K}_{\mathrm{s}}\left(\mathbf{q}_{\mathrm{e}, \mathrm{p}}\right)$ is defined by the identity ( $\mathrm{f}^{\prime}$ stands for the derivative with respect to $\mathrm{Re}$ )

$$
\bullet \mathbf{K}_{\mathrm{s}}\left(\mathbf{q}_{\mathrm{e}, \mathrm{p}}\right) \cdot \mathbf{q}_{\mathrm{e}, \mathrm{p}}^{\prime}=\mathbf{K}_{\mathrm{p}, \mathrm{s}} \cdot \mathbf{q}_{\mathrm{e}, \mathrm{p}}^{\prime}+\mathbf{N}_{\mathrm{s}}\left(\mathbf{q}_{\mathrm{e}, \mathrm{p}}^{\prime}\right) \cdot \mathbf{q}_{\mathrm{e}, \mathrm{p}}+\mathbf{N}_{\mathrm{s}}\left(\mathbf{q}_{\mathrm{e}, \mathrm{p}}\right) \cdot \mathbf{q}_{\mathrm{e}, \mathrm{p}}^{\prime},
$$

one obtains for $\mathbf{q}_{\mathrm{e}, \mathrm{p}}^{\prime}$ the following nonlinear $\left(\mathbf{K}_{\mathrm{s}}=\mathbf{K}_{\mathrm{s}}\left(\mathbf{q}_{\mathrm{e}, \mathrm{p}}\right)\right)$ differential equation:

$$
\bullet \mathbf{K}_{\mathrm{s}} \cdot \mathbf{q}_{\mathrm{e}, \mathrm{p}}^{\prime}+\mathbf{K}_{\mathrm{p}, \mathrm{s}}^{\prime} \cdot \mathbf{q}_{\mathrm{e}, \mathrm{p}}=\mathbf{F}_{\mathrm{p}, \mathrm{s}}^{\prime} .
$$
as

The solution of (2.16c) obviously exists and it is unique as long

$$
\operatorname{det} \mathbf{K}_{\mathrm{s}}\left(\mathbf{q}_{\mathrm{e}, \mathrm{p}}\right) \neq 0 \text {, }
$$

and if this latter condition is fulfilled one is able, by integrating (2.16c), to march the root of (2.16a) as the Reynolds number increases: equations $(2.16 \mathrm{c}, \mathrm{a})$ define a predictor-corrector method to determine the steady solution. In the other hand, if $\operatorname{det} \mathbf{K}_{\mathrm{s}}\left(\mathbf{q}_{\mathrm{e}, \mathrm{p}}\right)=$ 0 one would have a classic bifurcation of the equilibrium: the steady solution could then be marched out in Re by defining a proper ("statically stable") branch after the bifurcation. As it will be seen in the next item, the underlying assumption behind the asymptotic theory to be developed in this work implies in a condition stronger than (2.16d): within this context it can be taken here that a steady solution exists and it is uniquely defined.

If $\left\{\mathbf{x}_{\mathrm{k}} ; \mathrm{k}=1,2, \ldots, \mathrm{N}\right\}$ are the nodes of the Finite Element mesh then, given any continuous field $\mathbf{u}(\mathbf{x}, \mathbf{t})$, the "nodal interpolate" $\mathbf{u}_{\mathrm{h}}(\mathrm{x}, \mathrm{t})$ is defined by the expression

$\mathbf{u}_{\mathrm{h}}(\mathbf{x}, \mathrm{t})=\sum_{\mathrm{k}=1}^{\mathrm{N}}\left(\mathrm{u}\left(\mathbf{x}_{\mathrm{k}}, \mathrm{t}\right) \mathbf{i}+\mathrm{v}\left(\mathbf{x}_{\mathrm{k}}, \mathrm{t}\right) \mathbf{j}\right) \cdot \mathrm{h}_{\mathrm{k}}(\mathbf{x})=\sum_{\mathrm{k}=1}^{\mathrm{N}}\left(\mathrm{U}_{\mathrm{k}}(\mathrm{t}) \mathbf{i}+\mathrm{U}_{\mathrm{k}+\mathrm{N}}(\mathrm{t}) \mathbf{j}\right) \cdot \mathrm{h}_{\mathrm{k}}(\mathbf{x})$,

and, within the context of the discrete model, one can ignore the difference between $\mathbf{u}(\mathbf{x}, \mathrm{t})$ and its "nodal interpolate" $\mathbf{u}_{\mathrm{h}}(\mathbf{x}, \mathrm{t})$ : there is thus a one-to-one relation between the field $\mathbf{u}(\mathbf{x}, \mathbf{t})$ and the "nodal values vector" $\mathbf{U}(\mathrm{t})(\mathbf{u}(\mathbf{x}, \mathrm{t}) \Leftrightarrow \mathbf{U}(\mathrm{t}))$. If now $\mathbf{U}_{\mathrm{p}}$ is the "nodal values vector" of the auxiliary field $\mathbf{u}_{\mathrm{p}}(\mathbf{x})\left(\mathbf{u}_{\mathrm{p}}(\mathbf{x}) \Leftrightarrow \mathbf{U}_{\mathrm{p}}\right)$, the steady solution $\mathbf{u}_{\mathrm{e}}(\mathbf{x}) \Leftrightarrow \mathbf{U}_{\mathrm{e}}$ is defined by the expression

$$
\bullet \mathbf{U}_{\mathrm{e}}=\mathbf{U}_{\mathrm{p}}+\mathbf{T} \cdot \mathbf{q}_{\mathrm{e}, \mathrm{p}},
$$

and the global solution $\mathbf{u}_{\mathrm{T}}(\mathbf{x}, \mathrm{t}) \Leftrightarrow \mathbf{U}_{\mathrm{T}}(\mathrm{t})$ can be thus written as

$$
\text { - } \mathbf{U}_{\mathrm{T}}(\mathrm{t})=\mathbf{U}_{\mathrm{e}}+\mathbf{U}_{\mathrm{o}}(\mathrm{t}) ; \mathbf{U}_{\mathrm{o}}(\mathrm{t})=\mathbf{T} \cdot \mathbf{q}_{\mathrm{o}}(\mathrm{t}) .
$$

Keeping in mind these definitions and introducing also the field $\mathbf{u}_{\mathrm{o}}(\mathbf{x}, \mathrm{t}) \Leftrightarrow \mathbf{U}_{\mathrm{o}}(\mathrm{t})$, it is an easy task to show that the matrix $\mathbf{K}_{\mathrm{s}}$ can be expressed in the form (see $(2.5 b))$

$\bullet \int_{\mathrm{R}}\left[\left(\mathbf{u}_{\mathrm{e}} \cdot \nabla\right) \mathbf{u}_{\mathrm{o}}+\left(\mathbf{u}_{\mathrm{o}} \cdot \nabla\right) \mathbf{u}_{\mathrm{e}}\right] \cdot \delta \mathbf{u} \mathrm{dR}+\frac{1}{\mathrm{Re}_{\mathrm{R}}} \int_{\mathrm{R}}\left(\nabla \mathrm{u}_{\mathrm{o}} \cdot \nabla(\delta \mathrm{u})+\nabla \mathrm{v}_{\mathrm{o}} \cdot \nabla(\delta \mathrm{v})\right) \mathrm{dR}=\delta \mathbf{U}^{\mathrm{t}} \cdot \mathbf{K} \cdot \mathbf{U}_{\mathrm{o}}$ $\cdot \mathbf{K}_{\mathrm{s}}=\mathbf{T}^{\mathrm{t}} \cdot \mathbf{K} \cdot \mathbf{T}$.

The parcel $\left[\left(\mathbf{u}_{\mathrm{e}} \cdot \nabla\right) \mathbf{u}_{\mathrm{o}}+\left(\mathbf{u}_{\mathrm{o}} \cdot \nabla\right) \mathbf{u}_{\mathrm{e}}\right]$ of the convective acceleration introduces, as usual, a non-symmetry in $\mathbf{K}_{\mathrm{s}}$ that plays a role in stability theory to be addressed next.

\section{Hopf Bifurcation and Asymptotic Solution of Fluid Equation}

The differential equation for the perturbation $\mathbf{U}_{\mathrm{o}}(\mathrm{t})=\mathbf{T} \cdot \mathbf{q}_{\mathrm{o}}(\mathrm{t})$ on the steady solution $\mathbf{U}_{\mathrm{e}}$ can be easily derived by using the definition $\mathbf{q}(\mathrm{t})=\mathbf{q}_{\mathrm{e}, \mathrm{p}}+\mathbf{q}_{\mathrm{o}}(\mathrm{t})$ in (2.13); one obtains then, with the help of (2.16a), the homogeneous nonlinear equation

$$
\mathbf{M}_{\mathrm{s}} \cdot \dot{\mathbf{q}}_{\mathrm{o}}+\mathbf{K}_{\mathrm{s}} \cdot \mathbf{q}_{\mathrm{o}}+\mathbf{N}_{\mathrm{s}}\left(\mathbf{q}_{\mathrm{o}}\right) \cdot \mathbf{q}_{\mathrm{o}}=\mathbf{0}
$$

the non-symmetric matrix $\mathbf{K}_{\mathrm{s}}$ being defined in item (2.4). The eigenvalues of $\mathbf{K}_{\mathrm{s}}$ are thus complex, in general, and they will be defined as follows:

$$
\begin{aligned}
& \left\{\lambda_{1}=\sigma+i \omega ; \lambda_{2}=\sigma-i \omega ; \lambda_{3}=\sigma_{3}+i \omega_{3} ; \ldots ; \lambda_{\mathrm{s}}=\sigma_{\mathrm{s}}+\mathrm{i} \omega_{\mathrm{s}}\right\} \\
& \sigma \geq \sigma_{3} \geq \ldots \geq \sigma_{\mathrm{s}} .
\end{aligned}
$$

The first mode, the one that becomes first unstable since $\sigma \geq \sigma_{\mathrm{j}}$, is of the form

$$
\bullet \mathbf{q}_{\mathrm{o}}(\mathrm{t})=\mathrm{e}^{\lambda_{1} \mathrm{t}} \cdot \mathbf{E}
$$

where the mode $\mathbf{E}$ is such that $\left(\left(^{*}\right)\right.$ stands for the complex conjugate)

$$
\begin{aligned}
& \bullet\left(\lambda_{1} \mathbf{M}_{\mathrm{s}}+\mathbf{K}_{\mathrm{s}}\right) \cdot \mathbf{E}=\mathbf{0} ; \\
& \bullet\left(\mathbf{E}^{*}\right)^{\mathrm{t}} \cdot \mathbf{M}_{\mathrm{s}} \cdot \mathbf{E}=1 .
\end{aligned}
$$

Notice that $\lambda_{1}(\operatorname{Re})=\sigma(\operatorname{Re})+i \omega(\operatorname{Re})$ where, from $(2.19 b)$, it follows that

$$
\begin{aligned}
& \bullet \sigma(\mathrm{Re})=-1 / 2\left(\mathbf{E}^{*}\right)^{\mathrm{t}} \cdot\left(\mathbf{K}_{\mathrm{s}}+\mathbf{K}_{\mathrm{s}}^{\mathrm{t}}\right) \cdot \mathbf{E}=-1 / 2\left(\mathbf{T} \cdot \mathbf{E}^{*}\right)^{\mathrm{t}} \cdot\left(\mathbf{K}+\mathbf{K}^{\mathrm{t}}\right) \cdot(\mathbf{T} \cdot \mathbf{E}) \\
& \bullet i \omega(\operatorname{Re})=-1 / 2\left(\mathbf{E}^{*}\right)^{\mathrm{t}} \cdot\left(\mathbf{K}_{\mathrm{s}}-\mathbf{K}_{\mathrm{s}}^{\mathrm{t}}\right) \cdot \mathbf{E}=-1 / 2\left(\mathbf{T} \cdot \mathbf{E}^{*}\right)^{\mathrm{t}} \cdot\left(\mathbf{K}-\mathbf{K}^{\mathrm{t}}\right) \cdot(\mathbf{T} \cdot \mathbf{E}) .
\end{aligned}
$$

Relation (2.19c) can be used in conjunction to (2.17d) to provide more explicit expression for $\{\sigma(\operatorname{Re}) ; \omega(\operatorname{Re})\}$; indeed, if $\mathbf{e}(\mathbf{x}) \Leftrightarrow \mathbf{T} \cdot \mathbf{E}$ one obtains

$$
\begin{aligned}
& \bullet \sigma(R e)=-1 / 2 \int_{R}\left[u_{e}(\mathbf{x})\left(\frac{\partial \mathbf{e}}{\partial x} \cdot \mathbf{e}^{*}+\frac{\partial \mathbf{e}^{*}}{\partial x} \cdot \mathbf{e}\right)+v_{e}(\mathbf{x})\left(\frac{\partial \mathbf{e}}{\partial y} \cdot \mathbf{e}^{*}+\frac{\partial \mathbf{e}^{*}}{\partial y} \cdot \mathbf{e}\right)+2 \gamma_{\mathrm{e}}(\mathbf{x})\left(e_{x} e_{y}^{*}+e_{x}^{*} e_{y}\right)\right] d R- \\
& -1 / 2 \iint_{\mathrm{R}}\left(\frac{\partial \mathrm{u}_{\mathrm{e}}}{\partial \mathrm{x}}-\frac{\partial \mathrm{v}_{\mathrm{e}}}{\partial \mathrm{y}}\right) \cdot\left(\left|\mathrm{e}_{\mathrm{x}}\right|^{2}-\left|\mathrm{e}_{\mathrm{y}}\right|^{2}\right) \mathrm{dR}-\frac{1}{\mathrm{Re}_{\mathrm{R}}} \overbrace{\int_{\mathrm{R}}\left(\left|\nabla \mathrm{e}_{\mathrm{x}}\right|^{2}+\left|\nabla \mathrm{e}_{\mathrm{y}}\right|^{2}\right) \mathrm{dR}}^{\left.\mathrm{b}_{\mathrm{f}} \mathrm{Re}\right)}
\end{aligned}
$$

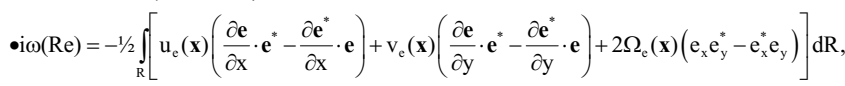

where $\gamma_{\mathrm{e}}(\mathbf{x})=1 / 2\left(\partial \mathrm{v}_{\mathrm{e}} / \partial \mathrm{x}+\partial \mathrm{u}_{\mathrm{e}} / \partial \mathrm{y}\right)$ is the shear rate of deformation of the steady solution $\mathbf{u}_{\mathrm{e}}(\mathbf{x})$ and $\Omega_{\mathrm{e}}(\mathbf{x})=1 / 2\left(\partial \mathrm{v}_{\mathrm{e}} / \partial \mathrm{x}-\partial \mathrm{u}_{\mathrm{e}} / \partial \mathrm{y}\right)$ its vorticity.

The above expression suggests to write $\sigma(\mathrm{Re})$ as $\sigma(\mathrm{Re})=a_{1}(\mathrm{Re})$ - $b_{1}(\mathrm{Re}) / \mathrm{Re}$, with $b_{1}(\mathrm{Re})>0$. The Strouhal number $\mathrm{S}(\mathrm{Re})^{5} \cong$ $\omega(\operatorname{Re}) / 2 \pi$ is known to change weakly with $\mathrm{Re}$ and from the structure of (2.20a) one should expect that both $\left\{a_{1}(\mathrm{Re}) ; b_{1}(\mathrm{Re})\right\}$ also do; if now one writes $\left\{a_{1}(\mathrm{Re})=a(\mathrm{Re}) \mathrm{S}(\mathrm{Re}) ; b_{1}(\mathrm{Re})=b(\mathrm{Re}) \cdot a(\mathrm{Re}) \mathrm{S}(\mathrm{Re})\right\}$ and assumes that $\left\{a_{0} ; \mathrm{S}_{0} ; b_{0}\right\}$ are typical values of the slowing varying functions $\{a(\mathrm{Re}) ; \mathrm{S}(\mathrm{Re}) ; b(\mathrm{Re})\}$ one obtains

$$
\cdot \sigma(\mathrm{Re})=a(\operatorname{Re}) \cdot \mathrm{S}(\mathrm{Re})\left[1-\frac{b(\mathrm{Re})}{\mathrm{Re}}\right] \cong a_{\mathrm{o}} \cdot \mathrm{S}_{\mathrm{o}}\left[1-\frac{b_{\mathrm{o}}}{\mathrm{Re}}\right],
$$

5 The actual Strouhal frequency $\omega s(\operatorname{Re})$ differs slightly from $\omega(\operatorname{Re})$, see (2.23b). 
an expression that has some empirical support, as discussed in item (2.6) below. It follows that $\sigma(\mathrm{Re})$ is (roughly) monotonically increasing with $\operatorname{Re}$, where $\sigma(\operatorname{Re})<0$ for $\operatorname{Re}<b_{\mathrm{o}}=\operatorname{Re}_{\mathrm{cr}}$ and $\sigma(\operatorname{Re})>$ 0 for $\operatorname{Re}>b_{\mathrm{o}}=\operatorname{Re}_{\mathrm{cr}}$. The value of $\operatorname{Re}_{\mathrm{cr}}$ inferred from numerical simulations seems to coalesce around $45\left(\operatorname{Re}_{\mathrm{cr}} \approx 45\right)$ although there are experimental evidences showing that this threshold value is a bit smaller $\left(\operatorname{Re}_{\mathrm{cr}} \approx 35\right)$. Obviously, for $\mathrm{Re}$ below $\mathrm{Re}_{\mathrm{cr}}$ the steady solution is stable $(\sigma(\operatorname{Re})<0)$ while it becomes unstable for $\mathrm{Re}>$ $\operatorname{Re}_{\text {cr }}(\sigma(\operatorname{Re})<0)$; furthermore, $\omega\left(\operatorname{Re}_{\text {cr }}\right) \neq 0$ and one has thus a typical Hopf bifurcation.

For $\mathrm{Re}$ above $\mathrm{Re}_{\mathrm{cr}}$ but close to it one has $0<\sigma(\operatorname{Re})<<1$, since $\sigma\left(\operatorname{Re}_{\mathrm{cr}}\right)=0$; furthermore, the experiments suggest - and the numerical results confirm - that only one mode is unstable in this range of Reynolds numbers $\left(\sigma_{\mathrm{j}}<0 ; \mathrm{j} \geq 3\right)$. The asymptotic solution to be developed is based on the following assumption

$$
\begin{aligned}
& \text { i) } 0<\sigma(\operatorname{Re})<<1 ; \omega(\operatorname{Re}) \cong \mathrm{O}(1) \text {; } \\
& \text { ii) } \sigma_{\mathrm{j}}(\operatorname{Re})<0 \text { for } \mathrm{j}=3, \ldots, \mathrm{s},
\end{aligned}
$$

that should be strictly satisfied in the vicinity of Hopf bifurcation; notice that (2.21) implies, necessarily, that $\left\{\lambda_{1}(\operatorname{Re}) \neq 0 ; \lambda_{\mathrm{j}}(\mathrm{Re}) \neq 0\right\}$, since $\left\{\omega(\operatorname{Re}) \neq 0 ; \sigma_{\mathrm{j}}(\operatorname{Re})<0\right\}$, and so $\operatorname{det} \mathbf{K}_{\mathrm{s}}(\mathrm{Re}) \neq 0$.

The adjoint eigenvalue problem $\left(\mathbf{K}_{\mathrm{s}} \rightarrow \mathbf{K}_{\mathrm{s}}^{\mathrm{t}}\right)$ plays a role, as it will be seen, in the derivation of the asymptotic theory. This problem has the same eigenvalues $(2.18 \mathrm{c})$ but the eigenvectors are distinct; in particular, to the "unstable" eigenvalue $\lambda_{1}$ it is associated the adjoint unstable mode $\mathbf{E}_{\mathrm{a}}$ where

$$
\bullet\left(\lambda_{1} \mathbf{M}_{\mathrm{s}}+\mathbf{K}_{\mathrm{s}}^{\mathrm{t}}\right) \cdot \mathbf{E}_{\mathrm{a}}=\mathbf{0} \cdot
$$

From (2.21) it follows that the "unstable" eigenvalue $\lambda_{1}=\sigma+$ $\mathrm{i} \omega$ is a single root of the related characteristic equation: if it were not, some of the $\lambda_{j}$ would be equal to $\lambda_{1}$ and the condition (ii) in (2.21) would be not fulfilled. Under the condition that $\lambda_{1}$ is a single root it is possible to show that

$$
\cdot \mathbf{E}_{\mathrm{a}}^{\mathrm{t}} \cdot \mathbf{M}_{\mathrm{s}} \cdot \mathbf{E} \neq 0,
$$

and thus $\mathbf{E}_{\mathrm{a}}$ can be normalized by the condition

$$
\bullet \mathbf{E}_{\mathrm{a}}^{\mathrm{t}} \cdot \mathbf{M}_{\mathrm{s}} \cdot \mathbf{E}=1,
$$

a relation that it will be used below in this section

The argument now is classic and it will be just sketched here: for $0<\sigma(\operatorname{Re})<<1$ the amplitude $A(t) \cdot e^{i \omega t}$ of the unstable mode $\mathbf{E}=$ $\mathbf{E}_{R}+\mathrm{i} \mathbf{E}_{I}$ increases (initially) exponentially with time $\left(\mathrm{A}(\mathrm{t}) \propto \mathrm{e}^{\sigma \mathrm{t}}\right.$; $\mathrm{dA} / \mathrm{dt}=\sigma \mathrm{A})$ and the solution of the dynamic system (2.18a) is attracted, since $\sigma_{j}<0$ for $j \geq 3$, to the (unstable) two dimensional manifold tangent to the plane generated by $\left\{\mathbf{E}_{\mathrm{R}} ; \mathbf{E}_{I}\right\}$; the exponential growth in this manifold is halted by the nonlinear term and expanding $\mathbf{N}\left(\mathbf{q}_{\mathrm{o}}\right) \cdot \mathbf{q}_{\mathrm{o}}$ in power series in the amplitude only the cubic term $-\mu|A(t)|^{2} A(t) \cdot e^{i \omega t}$ can match the term $\sigma A(t) \cdot e^{i \omega t}$ that causes the exponential growth. The equation for the amplitude $\mathrm{A}(\mathrm{t})$ - namely, Landau's equation - is thus given by ${ }^{6}$

6 It has been implicitly assumed here that one has a super-critical Hopf bifurcation with $\mu \mathrm{R}=$ Real $\mu>0$. This assumption has an experimental support, see Provansal \& Mathis \& Boyer (1987) and Leweke \& Provansal (1994) among others, and it has been also verified numerically by Noack \& Eckelmann (1994). Some preliminary numerical computation, to be published soon, corroborates this result.

$$
\bullet \frac{\mathrm{dA}}{\mathrm{dt}}-\sigma \mathrm{A}+\mu|\mathrm{A}|^{2} \mathrm{~A}=0 ; \mu=\mu_{\mathrm{R}}+\mathrm{i} \mu_{\mathrm{I}},
$$

the steady solution (limit cycle) $\mathrm{A}_{\mathrm{c}} \cdot \exp \left(\mathrm{i} \omega_{\mathrm{s}} \mathrm{t}\right)$ being given by

$$
\begin{aligned}
& \bullet \mathrm{A}_{\mathrm{c}}=\sqrt{\frac{\sigma}{\mu_{\mathrm{R}}}} ; \\
& \bullet \omega_{\mathrm{s}}=\omega-\frac{\mu_{\mathrm{I}}}{\mu_{\mathrm{R}}} \sigma .
\end{aligned}
$$

The formal asymptotic solution of the (discrete) Navier-Stokes equation will be derived next. In fact, writing the solution of (2.13) in the form

$$
\bullet \mathbf{q}(\mathrm{t})=\mathbf{q}_{\mathrm{e}, \mathrm{p}}+\mathbf{q}_{\mathrm{o}}(\mathrm{t})
$$

with the perturbation $\mathbf{q}_{\mathrm{o}}(\mathrm{t})$ satisfying $(2.18 \mathrm{a})$, one must have, to leading order, that $\mathbf{q}_{\mathrm{o}}(\mathrm{t}) \cong[\mathrm{A}(\mathrm{t}) \cdot \mathbf{E}+(*)]$, since the solution of (2.18a) should follow, at least initially, the unstable mode $\mathbf{E}$. The amplitude $\mathrm{A}(\mathrm{t})$, however, is such that (see (2.23))

$$
\begin{aligned}
& \cdot \mathrm{A}(\mathrm{t}) \cong \mathrm{O}\left(\sigma^{1 / 2}\right)<<1 ; \\
& \bullet \frac{\mathrm{dA}}{\mathrm{dt}} \cong \mathrm{O}(\sigma \mathrm{A}),
\end{aligned}
$$

and expanding $\mathbf{q}_{\mathrm{o}}(\mathrm{t})$ in the small parameter $\mathrm{A}(\mathrm{t}) \cong \mathrm{O}\left(\sigma^{1 / 2}\right)$ one obtains, with an error in $(2.24 \mathrm{a})$ of the form $\left[1+\mathrm{O}\left(\sigma^{2}\right)\right]$, that

$$
\begin{aligned}
\bullet \boldsymbol{q}_{\mathrm{o}}(\mathrm{t}) & =\underbrace{\left[\mathrm{A}(\mathrm{t}) \cdot \mathbf{E} \cdot \mathrm{e}^{\mathrm{i} \omega \mathrm{t}}+(*)\right]}_{\mathrm{O}\left(\sigma^{1 / 2}\right)}+\underbrace{\left[|\mathrm{A}(\mathrm{t})|^{2} \cdot \boldsymbol{\Lambda}_{20}+\left(\mathrm{A}^{2}(\mathrm{t}) \cdot \boldsymbol{\Lambda}_{22} \cdot \mathrm{e}^{2 \mathrm{i} \omega \mathrm{t}}+(*)\right)\right]}_{\mathrm{O}(\sigma)}+ \\
& +\underbrace{\left[|\mathrm{A}(\mathrm{t})|^{2} \mathrm{~A}(\mathrm{t}) \cdot \boldsymbol{\Lambda}_{31} \cdot \mathrm{e}^{\mathrm{i} \omega \mathrm{t}}+\mathrm{A}^{3}(\mathrm{t}) \cdot \boldsymbol{\Lambda}_{33} \cdot \mathrm{e}^{3 \mathrm{i} \omega t}+(*)\right]}_{\mathrm{O}\left(\sigma^{3 / 2}\right)}+\mathrm{O}\left(\sigma^{2}\right) .
\end{aligned}
$$

The nonlinear term in (2.18a) can, accordingly, be written as

$\cdot \mathbf{N}_{\mathrm{s}}\left(\mathbf{q}_{\mathrm{o}}\right) \cdot \mathbf{q}_{\mathrm{o}}=\left[\mathbf{N}_{20}+\left(\mathbf{N}_{22} \cdot \mathrm{e}^{2 \mathrm{i \omega t}}+(*)\right)\right]+\left[\mathbf{N}_{31} \cdot \mathrm{e}^{\mathrm{i} \omega t}+\mathbf{N}_{33} \cdot \mathrm{e}^{3 i \omega t}+\left(^{*}\right)\right]+\mathrm{O}\left(\sigma^{2}\right)$

with

$\bullet \mathbf{N}_{20}=\mathbf{N}_{\mathrm{s}}(\mathbf{E}) \cdot \mathbf{E}^{*}+\mathbf{N}_{\mathrm{s}}\left(\mathbf{E}^{*}\right) \cdot \mathbf{E}$;

$\bullet \mathbf{N}_{22}=\mathbf{N}_{\mathrm{s}}(\mathbf{E}) \cdot \mathbf{E}$;

$\bullet \mathbf{N}_{31}=\mathbf{N}_{\mathrm{s}}(\mathbf{E}) \cdot \boldsymbol{\Lambda}_{20}+\mathbf{N}_{\mathrm{s}}\left(\boldsymbol{\Lambda}_{20}\right) \cdot \mathbf{E}+\mathbf{N}_{\mathrm{s}}\left(\boldsymbol{\Lambda}_{22}\right) \cdot \mathbf{E}^{*}+\mathbf{N}_{\mathrm{s}}\left(\mathbf{E}^{*}\right) \cdot \boldsymbol{\Lambda}_{22} ;$

$\bullet \mathbf{N}_{33}=\mathbf{N}_{\mathrm{s}}(\mathbf{E}) \cdot \boldsymbol{\Lambda}_{22}+\mathbf{N}_{\mathrm{s}}\left(\boldsymbol{\Lambda}_{22}\right) \cdot \mathbf{E}$.

Placing (2.25) into (2.18a) one obtains $\left(\lambda_{1}=\sigma+i \omega\right)$

$\left[\left(\frac{\mathrm{dA}}{\mathrm{dt}}-\sigma \mathrm{A}\right) \mathbf{M}_{\mathrm{s}} \cdot \mathbf{E}+|\mathrm{A}|^{2} \mathrm{~A}\left(\mathbf{N}_{31}+\left(\lambda_{1} \mathbf{M}_{\mathrm{s}}+\mathbf{K}_{\mathrm{s}}\right) \cdot \boldsymbol{\Lambda}_{31}\right)\right] \mathrm{e}^{\mathrm{i} \omega \mathrm{t}}+$

$+|\mathrm{A}|^{2}\left[\mathbf{K}_{\mathrm{s}} \cdot \boldsymbol{\Lambda}_{20}+\mathbf{N}_{20}\right]+\mathrm{A}^{2}\left[\left(2 \mathrm{i} \omega \mathbf{M}_{\mathrm{s}}+\mathbf{K}_{\mathrm{s}}\right) \boldsymbol{\Lambda}_{22}+\mathbf{N}_{22}\right] \mathrm{e}^{2 \mathrm{i \omega t}}+{ }^{(2.26 \mathrm{a})}$

$+\mathrm{A}^{3}\left[\left(3 \mathrm{i} \omega \mathbf{M}_{\mathrm{s}}+\mathbf{K}_{\mathrm{s}}\right) \boldsymbol{\Lambda}_{33}+\mathbf{N}_{33}\right] \mathrm{e}^{3 \mathrm{i} \omega \mathrm{t}}+\mathrm{O}\left(\sigma^{2}\right)=0$, 
where the term $|\mathrm{A}|^{2} \mathrm{~A}\left(\sigma \mathbf{M}_{\mathrm{s}} \cdot \Lambda_{31}\right) \mathrm{e}^{\mathrm{i} \omega t} \cong \mathrm{O}\left(\sigma^{5 / 2}\right)$ has been added just for convenience; if now $\left\{\Lambda_{20} ; \Lambda_{22} ; \Lambda_{33}\right\}$ are solutions of the (nonsingular $\left.{ }^{7}\right)$ linear systems

$$
\begin{aligned}
& \cdot \mathbf{K}_{\mathrm{s}} \cdot \boldsymbol{\Lambda}_{20}+\mathbf{N}_{20}=\mathbf{0} ; \\
& \bullet\left(2 \mathrm{i} \omega \mathbf{M}_{\mathrm{s}}+\mathbf{K}_{\mathrm{s}}\right) \cdot \boldsymbol{\Lambda}_{22}+\mathbf{N}_{22}=\mathbf{0} ; \\
& \bullet\left(3 \mathrm{i} \omega \mathbf{M}_{\mathrm{s}}+\mathbf{K}_{\mathrm{s}}\right) \cdot \boldsymbol{\Lambda}_{33}+\mathbf{N}_{33}=\mathbf{0},
\end{aligned}
$$

(2.26a) reduces to

$$
\left(\frac{\mathrm{dA}}{\mathrm{dt}}-\sigma \mathrm{A}\right) \mathbf{M}_{\mathrm{s}} \cdot \mathbf{E}+|\mathrm{A}|^{2} \mathrm{~A}\left(\mathbf{N}_{31}+\left(\lambda_{1} \mathbf{M}_{\mathrm{s}}+\mathbf{K}_{\mathrm{s}}\right) \cdot \boldsymbol{\Lambda}_{31}\right)=0 \cdot
$$

Multiplying (2.26c) on the left by the adjoint unstable mode $\mathbf{E}_{\mathrm{a}}$ and using $(2.22 \mathrm{a}, \mathrm{c})$ Landau's equation $(2.23 \mathrm{a})$ is obtained with

$$
\bullet \mu \mathbf{E}_{\mathrm{a}}^{\mathrm{t}} \cdot \mathbf{N}_{31} .
$$

Summarizing: solving the eigenvalues problems (2.19b) and (2.22a,c) the vectors $\left\{\mathbf{N}_{20} ; \mathbf{N}_{22}\right\}$ can be computed from (2.25c) and the solutions $\left\{\Lambda_{20} ; \Lambda_{22}\right\}$ of the linear systems (2.26b) can be determined; with them the vector $\mathbf{N}_{31}$ can be obtained from (2.25c) and Landau's coefficient $\mu$ is thus given by (2.27).

Writing now $\mathbf{N}_{31}$ in the form

$$
\begin{aligned}
& \mathbf{N}_{31}=\mu \mathbf{M}_{\mathrm{s}} \cdot \mathbf{E}+\mathbf{N}_{31, \perp}^{(\mathrm{a})} ; \\
& \mathbf{E}_{\mathrm{a}}^{\mathrm{t}} \cdot \mathbf{N}_{31, \perp}^{(\mathrm{a})}=0,
\end{aligned}
$$

and placing (2.28a) into (2.26c) one obtains, with the help of (2.23a), the equality

$$
\delta \mathbf{q}^{\mathrm{t}} \cdot\left[\left(\lambda_{1} \mathbf{M}_{\mathrm{s}}+\mathbf{K}_{\mathrm{s}}\right) \cdot \boldsymbol{\Lambda}_{31}+\mathbf{N}_{31, \perp}^{(\mathrm{a})}\right]=0 \text { all } \delta \mathbf{q} \in \mathrm{J}_{\mathrm{s}} .
$$

Introducing the (s -2$)$-dimensional sub-spaces $\left\{\mathrm{J}_{\perp} ; \mathrm{J}_{\mathrm{a}, \perp}\right\}$ of $\mathrm{J}_{\mathrm{s}}$ by the definitions

$$
\begin{aligned}
& \bullet \mathrm{J}_{\perp}=\left\{\mathbf{q}_{\perp}: \mathbf{q}_{\perp}^{\mathrm{t}} \cdot \mathbf{E}=0\right\} \Rightarrow \mathbf{q}_{\perp}=\mathbf{S}_{\perp} \cdot \mathbf{x} ; \\
& \bullet \mathrm{J}_{\mathrm{a}, \perp}=\left\{\mathbf{q}_{\mathrm{a}, \perp}: \mathbf{q}_{\mathrm{a}, \perp}^{\mathrm{t}} \cdot \mathbf{E}_{\mathrm{a}}=0\right\} \Rightarrow \mathbf{q}_{\mathrm{a}, \perp}=\mathbf{S}_{\mathrm{a}, \perp} \cdot \mathbf{x},
\end{aligned}
$$

with $\mathbf{x}$ being an arbitrary ( $\mathrm{s}-2)$-dimensional vector, and the matrices

$$
\begin{aligned}
& \bullet \mathbf{M}_{\mathrm{s}, \perp}=\mathbf{S}_{\mathrm{a}, \perp}^{\mathrm{t}} \cdot \mathbf{M}_{\mathrm{s}} \cdot \mathbf{S}_{\perp} ; \\
& \bullet \mathbf{K}_{\mathrm{s}, \perp}=\mathbf{S}_{\mathrm{a}, \perp}^{\mathrm{t}} \cdot \mathbf{K}_{\mathrm{s}} \cdot \mathbf{S}_{\perp},
\end{aligned}
$$

the vector $\Lambda_{31}$ can be obtained from the solution of the (non singular $\left.{ }^{8}\right)$ linear system

$$
\begin{aligned}
& \bullet\left(\lambda_{1} \mathbf{M}_{\mathrm{s}, \perp}+\mathbf{K}_{\mathrm{s}, \perp}\right) \cdot \mathbf{x}_{31}+\mathbf{S}_{\mathrm{a}, \perp}^{\mathrm{t}} \cdot \mathbf{N}_{31, \perp}^{(\mathrm{a})}=\mathbf{0} ; \\
& \bullet \boldsymbol{\Lambda}_{31}=\mathbf{S}_{\perp} \cdot \mathbf{x}_{31} .
\end{aligned}
$$

Reverting to the "nodal values vector" $\mathbf{U}(\mathrm{t})=\mathbf{T} \cdot\left(\mathbf{q}_{\mathrm{e}, \mathrm{p}}+\mathbf{q}_{\mathrm{o}}(\mathrm{t})\right)$, see (2.1c), (2.17), one has

7 Notice that (2.21) rules out $\{0 ; 2 i \omega ; 3 i \omega\}$ as possible eigenvalues of Ks once $\sigma \mathrm{j}$ $<0$ for $\mathrm{j} \geq 3$ and $\omega \neq 0$; the matrices $\{\mathrm{Ks} ;(2 \mathrm{i} \omega \mathrm{Ms}+\mathrm{Ks}) ;(3 \mathrm{i} \omega \mathrm{Ms}+\mathrm{Ks})\}$ in $(2.26 \mathrm{~b})$ are thus non-singular.

8 Recall that (2.21) implies that $\lambda 1$ is a single root of the characteristic polynomial of $\mathrm{Ks}$ and so the matrix $\lambda 1 \mathrm{Ms}+\mathrm{Ks}$, restricted to the sub-space orthogonal to the eigenvectors $\{\mathrm{E} ; \mathrm{Ea}\}$, must be non-singular.

$$
\begin{aligned}
\cdot \mathbf{U}(\mathrm{t}) & =\mathbf{U}_{\mathrm{e}, \mathrm{p}}+\underbrace{\left[\mathrm{A}(\mathrm{t}) \cdot \mathbf{E}_{\mathrm{U}} \cdot \mathrm{e}^{\mathrm{i} \omega \mathrm{t}}+(*)\right]}_{O\left(\sigma^{1 / 2}\right)}+\underbrace{\left[|\mathrm{A}(\mathrm{t})|^{2} \cdot \boldsymbol{\Lambda}_{20, \mathrm{U}}+\left(\mathrm{A}^{2}(\mathrm{t}) \cdot \boldsymbol{\Lambda}_{22, \mathrm{U}} \cdot \mathrm{e}^{2 i \omega t}+(*)\right)\right]}_{\mathrm{O}(\sigma)}+ \\
& +\underbrace{\left[|\mathrm{A}(\mathrm{t})|^{2} \mathrm{~A}(\mathrm{t}) \cdot \boldsymbol{\Lambda}_{31, \mathrm{U}} \cdot \mathrm{e}^{\mathrm{i \omega t}}+\mathrm{A}^{3}(\mathrm{t}) \cdot \boldsymbol{\Lambda}_{33, \mathrm{U}} \cdot \mathrm{e}^{3 i \omega t}+(*)\right]}_{O\left(\sigma^{3 / 2}\right)}+\mathrm{O}\left(\sigma^{2}\right),
\end{aligned}
$$

with

$$
\bullet\left\{\mathbf{U}_{\mathrm{e}, \mathrm{p}} ; \mathbf{E}_{\mathrm{U}} ; \boldsymbol{\Lambda}_{20, \mathrm{U}} ; \boldsymbol{\Lambda}_{22, \mathrm{U}} ; \boldsymbol{\Lambda}_{31, \mathrm{U}} ; \boldsymbol{\Lambda}_{33, \mathrm{U}}\right\}=\mathbf{T} \cdot\left\{\mathbf{q}_{\mathrm{e}, \mathrm{p}} ; \mathbf{E} ; \boldsymbol{\Lambda}_{20} ; \boldsymbol{\Lambda}_{22} ; \boldsymbol{\Lambda}_{31} ; \boldsymbol{\Lambda}_{33}\right\}
$$

and placing (2.29a) into (2.14a) one obtains

$$
\begin{aligned}
\cdot \mathbf{F}_{\mathrm{D}}(\mathrm{t}) & =\mathbf{F}_{00}+\underbrace{\left[\mathrm{A}(\mathrm{t}) \cdot \mathbf{F}_{11} \cdot \mathrm{e}^{\mathrm{i} \omega \mathrm{t}}+\left(^{*}\right)\right]}_{\mathrm{O}\left(\sigma^{1 / 2}\right)}+\underbrace{\left[|\mathrm{A}(\mathrm{t})|^{2} \cdot \mathbf{F}_{20}+\left(\mathrm{A}^{2}(\mathrm{t}) \cdot \mathbf{F}_{22} \cdot \mathrm{e}^{2 \mathrm{i} \omega \mathrm{t}}+(*)\right)\right]}_{O(\sigma)}+ \\
+ & \underbrace{\left[|\mathrm{A}(\mathrm{t})|^{2} \mathrm{~A}(\mathrm{t}) \cdot \mathbf{F}_{31} \cdot \mathrm{e}^{\mathrm{i} \omega t}+\mathrm{A}^{3}(\mathrm{t}) \cdot \mathbf{F}_{33} \cdot \mathrm{e}^{3 i \omega t}+\left({ }^{*}\right)\right]}_{O\left(\sigma^{3 / 2}\right)}+\mathrm{O}\left(\sigma^{2}\right),
\end{aligned}
$$

with (see (2.17d) and (2.23a) with $\left.\lambda_{1}=\sigma+i \omega\right)$

$$
\begin{aligned}
& \cdot \mathbf{F}_{00}=\mathbf{K}_{\mathrm{p}} \cdot \mathbf{U}_{\mathrm{e}, \mathrm{p}}+\mathbf{N}\left(\mathbf{U}_{\mathrm{e}, \mathrm{p}}\right) \cdot \mathbf{U}_{\mathrm{e}, \mathrm{p}}-\mathbf{F}_{\mathrm{p}} ; \\
& \cdot \mathbf{F}_{11}=\left(\lambda_{1} \mathbf{M}+\mathbf{K}\right) \cdot \mathbf{E}_{\mathrm{U}} ; \\
& \cdot \mathbf{F}_{20}=\mathbf{K} \cdot \boldsymbol{\Lambda}_{20, \mathrm{U}}+\mathbf{N}_{20, \mathrm{U}} ; \\
& \bullet \mathbf{F}_{22}=(2 \mathrm{i} \omega \mathbf{M}+\mathbf{K}) \cdot \boldsymbol{\Lambda}_{22, \mathrm{U}}+\mathbf{N}_{22, \mathrm{U}} ; \\
& \cdot \mathbf{F}_{31}=\left(\lambda_{1} \mathbf{M}+\mathbf{K}\right) \cdot \boldsymbol{\Lambda}_{31, \mathrm{U}}-\mu \mathbf{M} \cdot \mathbf{E}_{\mathrm{U}}+\mathbf{N}_{31, \mathrm{U}} ; \\
& \cdot \mathbf{F}_{33}=(3 i \omega \mathbf{M}+\mathbf{K}) \cdot \boldsymbol{\Lambda}_{33, \mathrm{U}}+\mathbf{N}_{33, \mathrm{U}},
\end{aligned}
$$

where $\left\{\mathbf{N}_{20, \mathrm{U}} ; \mathbf{N}_{22, \mathrm{U}} ; \mathbf{N}_{31, \mathrm{U}} ; \mathbf{N}_{33, \mathrm{U}}\right\}$ are defined as in (2.25c) with $\left\{\mathbf{N}_{\mathrm{jk}} \rightarrow \mathbf{N}_{\mathrm{jk}, \mathrm{U}} ; \mathbf{E} \rightarrow \mathbf{E}_{\mathrm{U}} ; \Lambda_{20} \rightarrow \Lambda_{20, \mathrm{U}} ; \Lambda_{22} \rightarrow \Lambda_{22, \mathrm{U}}\right\}$. Considering now the solutions of the Poisson's equations (see (2.14c))

$$
\bullet \boldsymbol{\Delta} \cdot \mathbf{P}_{\mathrm{jk}}=\mathbf{R}^{\mathrm{t}} \cdot \mathbf{F}_{\mathrm{jk}} ;(\mathrm{jk})=\{(00) ;(11) ;(20) ;(22) ;(31) ;(33)\},
$$

the (discrete) pressure field is given by $\left(\mathbf{P}_{\mathrm{e}}=\mathbf{P}_{00}\right.$ is the pressure related to $\left.\mathbf{u}_{\mathrm{e}}(\mathbf{x}) \Leftrightarrow \mathbf{U}_{\mathrm{e}}\right)$

$$
\begin{aligned}
\cdot \mathbf{P}(\mathrm{t}) & =\mathbf{P}_{\mathrm{e}}+\underbrace{\left[\mathrm{A}(\mathrm{t}) \cdot \mathbf{P}_{11} \cdot \mathrm{e}^{\mathrm{i} \omega \mathrm{t}}+(*)\right]}_{\mathrm{O}\left(\mathrm{\sigma}^{1 / 2}\right)}+\underbrace{\left[|\mathrm{A}(\mathrm{t})|^{2} \cdot \mathbf{P}_{20}+\left(\mathrm{A}^{2}(\mathrm{t}) \cdot \mathbf{P}_{22} \cdot \mathrm{e}^{2 i \omega t}+(*)\right)\right]}_{\mathrm{O}(\sigma)}+ \\
& +\underbrace{\left[|\mathrm{A}(\mathrm{t})|^{2} \mathrm{~A}(\mathrm{t}) \cdot \mathbf{P}_{31} \cdot \mathrm{e}^{\mathrm{i} \omega \mathrm{t}}+\mathrm{A}^{3}(\mathrm{t}) \cdot \mathbf{P}_{33} \cdot \mathrm{e}^{3 \mathrm{i} \omega t}+(*)\right]}_{O\left(\sigma^{3 / 2}\right)}+\mathrm{O}\left(\sigma^{2}\right),
\end{aligned}
$$

with the same error factor $\left[1+\mathrm{O}\left(\sigma^{2}\right)\right]$ of the velocity field approximation

Expressions (2.29a) and (2.30d) synthesize the asymptotic solution of the (discrete) Navier-Stokes equation in the vicinity of Hopf bifurcation $\left(\mathrm{Re} \approx \mathrm{Re}_{\mathrm{cr}}\right.$ ); in the next item, the possibility to extend this solution to the range $\mathrm{Re}>>\mathrm{Re}_{\mathrm{cr}}$ is discussed.

\section{Extension of Landau's Equation Beyond Hopf Bifurcation}

The asymptotic solution of the (discrete) Navier-Stokes was derived assuming (2.21), two conditions that hold in the vicinity of the Hopf bifurcation but not necessarily only there. The purpose here is to give arguments that suggest that (2.21) - and, with it, the given asymptotic solution - can be extended far beyond Hopf bifurcation, that is, to the range $\operatorname{Re}>>\mathrm{Re}_{\mathrm{cr}}$. 
In fact, numerical results by Noack \& Eckelmann (1994) indicate that (2.21) remains essentially correct in the range $\mathrm{Re}_{\mathrm{cr}} \cong 45$ $<\operatorname{Re}<300$ and Henderson (1997), after a detailed numerical work, affirms that up to $\operatorname{Re}=1000$ no other bifurcation, besides the one at $\mathrm{Re}_{\mathrm{cr}}$, could be observed in the two dimensional model; or, in other words, that $\left\{\sigma_{j}<0 ; j \geq 3\right\}$ in this range.

From the experimental side, Provansal \& Mathis \& Boyer (1987) have observed that the inferred value of $\sigma(\mathrm{Re})$ could be fitted to the expression

$$
\bullet \sigma(\mathrm{Re})=\frac{\sigma(\mathrm{Re}) d}{\mathrm{U}} \square 0.20\left(1-\frac{\mathrm{Re}_{\mathrm{cr}}}{\mathrm{Re}}\right),
$$

while Leweke \& Provansal assumed (2.31) in the range $\operatorname{Re}_{\text {cr }} \cong 45<$ $\operatorname{Re}<300$.

As already discussed, the empirical relation (2.31) seems to have a foot on the more basic set of equations that describe the fluid flow, since a similar expression can be derived exactly, see (2.20b); furthermore, it indicates that $\sigma(\mathrm{Re})$ can be considered a "small parameter" of order 0.20 (or less). All together, these evidences suggest that (2.21) could be pushed at least up to $\mathrm{Re} \approx 1000$ since, following Henderson, no other bifurcation (in 2D) could be found in this range while (2.31), together with (2.20b), seems to indicate that $\sigma(\mathrm{Re})$ remains in fact "small" as asserted in (2.21).

Experimental results on VIV are mostly in the range $10^{3}<\mathrm{Re}<$ $10^{4}$, see Khalak \& Wiliamson (1996), and they do not seem to depend very much on Re. The observed harmonic pattern is very neat and this, undoubtedly, was the motivation behind a bold assumption introduced by Bishop \& Hassan (1964) to describe the flow around a circular cylinder: they proposed to represent the flow by an one degree of freedom "wake oscillator model" based on Van der Pol equation that leads, for a small enough $\sigma$, to Landau's equation (2.23a); this idea was further developed and it is the basis of the so-called phenomenological models used to predict VIV, see Iwan \& Blevins (1975). In despite of the loose link with the more basic flow equation the predictions from these models have some accuracy, showing that the ordered oscillatory behavior in the wake can be apparently described by means of an one degree of freedom system related to the unstable mode of the problem. The smallness of $\sigma$, a common feature in all "wake oscillator models", coupled to the flow representation by only one unstable mode, can be translated in the following words: the basic assumption (2.21) is, apparently, correct in the range $10^{3}<\mathrm{Re}<10^{4}$ of Reynolds numbers of the VIV experiments. However, from $\mathrm{Re} \approx 10^{4}$ until the transition region $\mathrm{Re}$ $\approx 10^{5}$ nothing very much different occurs and one can possibly push (2.21) up to the transition region $\mathrm{Re} \approx 10^{5}$. For $\mathrm{Re}>10^{6}$ the boundary layer is fully turbulent but a (relatively) well defined Strouhal frequency can be detected again: in line with the overall view taken here, one speculates that the same conditions (2.21) can hold if one searches for the stability of the time averaged (turbulent ${ }^{9}$ ) symmetric solution of the flow around the circular cylinder.

These extensions should be obviously confirmed numerically but certainly the present theory has a range of application much broader than foreseen a priori; more than that, $(2.21)$ can be used to determine precisely this range.

\section{Weak Three-Dimensionality: Ginzburg-Landau Equation}

The perturbation $\mathbf{u}_{\mathrm{o}}(\mathbf{x}, \mathrm{t})$ on the $2 \mathrm{D}$ steady solution $\mathbf{u}_{\mathrm{e}}(\mathbf{x})$ is, to leading order, given by $\mathbf{u}_{\mathrm{o}}(\mathbf{x}, \mathrm{t}) \cong \mathrm{A}(\mathrm{t}) \cdot \mathbf{e}(\mathbf{x})$ where $\mathbf{e}(\mathbf{x}) \Leftrightarrow \mathbf{T} \cdot \mathbf{E}$ is the

9 Boundary layer turbulence is likely due to the concomitant instabilities of several symmetric modes, as elaborated in a forthcoming paper; it will be also discussed there a possible scenario for the transition region $105<\operatorname{Re}<106$. unstable mode and $\mathrm{A}(\mathrm{t})$ its complex amplitude: $\mathrm{A}(\mathrm{t})=$ $|A(t)| \cdot \exp (\varphi(t))$. It is known for a long time - see, for instance, Toebes (1969) - that the vortices are not shed in phase along the span of a fixed cylinder, and in fact the correlation among the vortices emitted in distinct sections tends to zero "very fast"10, in a length of order of $10 \mathrm{~d}$. The phase $\varphi$ should then change in the longitudinal $\mathrm{z}$-direction and so it does the amplitude $\mathrm{A}$ and the perturbation $\mathbf{u}_{\mathrm{o}}(\mathbf{x}, \mathrm{t})$; or $\left\{\mathrm{A}=\mathrm{A}(\mathrm{z}, \mathrm{t}) ; \mathbf{u}_{\mathrm{o}}=\mathbf{u}_{\mathrm{o}}(\mathbf{x}, \mathrm{z}, \mathrm{t})\right\}$, where $\mathbf{x}=\mathrm{xi}+$ $y \mathbf{j}$ continues to represent the position vector in the cross section plane and $\mathbf{u}_{0}(\mathbf{x}, \mathrm{z}, \mathrm{t})=\mathrm{u}_{\mathrm{o}}(\mathbf{x}, \mathrm{z}, \mathrm{t}) \mathbf{i}+\mathrm{v}_{\mathrm{o}}(\mathbf{x}, \mathrm{z}, \mathrm{t}) \mathbf{j}$ the perturbed velocity field in this plane at the z-level. The longitudinal component of the perturbed velocity field will be designated by $\mathrm{w}_{\mathrm{o}}(\mathbf{x}, \mathrm{z}, \mathrm{t})$.

The variation of $\mathrm{A}(\mathrm{z}, \mathrm{t})$ in the longitudinal $\mathrm{z}$-direction should be expressed by an even derivative with respect to $z$, since there is no preferred direction, and observing that the viscous diffusion in this direction, given by $(1 / \operatorname{Re}) \partial^{2} \mathbf{u}_{\mathrm{o}} / \partial \mathrm{z}^{2}$, implies to leading order in a term proportional to $\partial^{2} \mathrm{~A} / \partial \mathrm{z}^{2}$, the following $3 \mathrm{D}$ correction is proposed for Landau's equation (2.23a):

$$
\bullet \frac{\partial \mathrm{A}}{\partial \mathrm{t}}-\sigma \mathrm{A}-\gamma \frac{\partial^{2} \mathrm{~A}}{\partial \mathrm{z}^{2}}+\mu|\mathrm{A}|^{2} \mathrm{~A}=0 .
$$

This is the Ginzburg-Landau Equation (GLE), first proposed by Ginzburg more than fifty years ago in his study on superconductivity, see Ginzburg \& Landau (1950); notice that, in general, both Landau's coefficient $\mu$ and Ginzburg's coefficient $\gamma$ are complex numbers: $\left\{\mu=\mu_{R}+i \mu_{\mathrm{I}} ; \gamma=\gamma_{\mathrm{R}}+\mathrm{i} \gamma_{\mathrm{I}}\right\}$. Assuming, as before, $\sigma<<1$ (see (2.21)) and recalling that $\mathrm{A} \cong \mathrm{O}\left(\sigma^{1 / 2}\right)$, a proper balance of the terms in (3.1a) indicates that the length scale $l_{\mathrm{z}}$ for the longitudinal variation of $\mathrm{A}(\mathrm{z}, \mathrm{t})$ must be such that

$$
\bullet l_{\mathrm{z}} \cong \mathrm{O}\left(\frac{d}{\sqrt{\sigma}}\right)>>d,
$$

or, in short: (3.1a) deals with a weak three-dimensional variation of the flow field. As it is discussed in the last item of the present section, GLE can be easily extended to the case where both the geometry and the incident flow change in the longitudinal direction if the rate of change is weaker than (3.1b).

In what follows, (3.1a) will be obtained as a consistent asymptotic approximation of the NSE and, in deriving it, a procedure to determine Ginzburg's coefficient $\gamma$ will be defined.

\section{Asymptotic Approximation for 3D Field Equation}

Let $\mathbf{u}_{(3 \mathrm{~d})}(\mathbf{x}, \mathrm{z}, \mathrm{t})=\mathbf{u}_{\mathrm{e}}(\mathbf{x})+\left[\mathbf{u}_{\mathrm{o}}(\mathbf{x}, \mathrm{z}, \mathrm{t})+\mathrm{w}_{\mathrm{o}}(\mathbf{x}, \mathrm{z}, \mathrm{t}) \mathbf{k}\right]$ be the $3 \mathrm{D}$ velocity field for the flow around a slender cylinder, with $\mathbf{u}_{\mathrm{e}}(\mathbf{x})$ being the $2 \mathrm{D}$ steady solution and $\left[\mathbf{u}_{\mathrm{o}}(\mathbf{x}, \mathrm{z}, \mathrm{t})+\mathrm{w}_{\mathrm{o}}(\mathbf{x}, \mathrm{z}, \mathrm{t}) \mathbf{k}\right]$ the perturbation on it; if, as defined in section (2), $\nabla=\mathbf{i} \partial / \partial \mathbf{x}+\mathbf{j} \partial / \partial \mathrm{y}$, the 3D NSE for the perturbation $\left[\mathbf{u}_{\mathrm{o}}(\mathbf{x}, \mathrm{z}, \mathrm{t})+\mathrm{w}_{\mathrm{o}}(\mathbf{x}, \mathrm{z}, \mathrm{t}) \mathbf{k}\right]$ is given by

$$
\begin{aligned}
& \bullet \frac{\partial \mathbf{u}_{\mathrm{o}}}{\partial \mathrm{t}}+\left(\mathbf{u}_{\mathrm{e}} \cdot \nabla\right) \mathbf{u}_{\mathrm{o}}+\left(\mathbf{u}_{\mathrm{o}} \cdot \nabla\right) \mathbf{u}_{\mathrm{e}}+\left(\mathbf{u}_{\mathrm{o}} \cdot \nabla\right) \mathbf{u}_{\mathrm{o}}-\frac{1}{\mathrm{Re}} \nabla^{2} \mathbf{u}_{\mathrm{o}}+\nabla \mathrm{p}_{\mathrm{o}}=\underbrace{\frac{1}{\mathrm{Re}} \frac{\partial^{2} \mathbf{u}_{\mathrm{o}}}{\partial \mathrm{z}^{2}}}_{\mathrm{O}\left(\sigma^{3 / 2}\right)}-\underbrace{\mathrm{w}_{\mathrm{o}} \frac{\partial \mathbf{u}_{\mathrm{o}}}{\partial \mathrm{z}}}_{\mathrm{O}\left(\sigma \mathrm{w}_{\mathrm{o}}\right)} ; \\
& \bullet \underbrace{\frac{\partial \mathrm{w}_{\mathrm{o}}}{\partial \mathrm{t}}+\left(\mathbf{u}_{\mathrm{e}} \cdot \nabla\right) \mathrm{w}_{\mathrm{o}}-\frac{1}{\mathrm{Re}} \nabla^{2} \mathrm{w}_{\mathrm{o}}}_{\mathrm{O}\left(\mathrm{w}_{\mathrm{o}}\right)}=-\underbrace{\frac{\partial \mathrm{p}_{\mathrm{o}}}{\partial \mathrm{z}}}_{\mathrm{O}(\sigma)}-\underbrace{\left(\mathbf{u}_{\mathrm{o}} \cdot \nabla\right) \mathrm{w}_{\mathrm{o}}}_{\mathrm{O}\left(\sigma^{1 / 2} \mathrm{w}_{\mathrm{o}}\right)}-\underbrace{\mathrm{w}_{\mathrm{o}} \frac{\partial \mathrm{w}_{\mathrm{o}}}{\partial \mathrm{z}}}_{\mathrm{O}\left(\sigma^{1 / 2} \mathrm{w}_{\mathrm{o}}^{2}\right)}+\underbrace{\frac{1}{\mathrm{Re} \frac{\partial^{2} \mathrm{w}_{\mathrm{o}}}{\partial \mathrm{z}^{2}}}}_{\mathrm{O}\left(\sigma \mathrm{w}_{\mathrm{o}}\right)} ; \\
& \bullet \nabla \cdot \mathbf{u}_{\mathrm{o}}=-\underbrace{\frac{\partial \mathrm{w}_{\mathrm{o}}}{\partial \mathrm{z}}}_{\mathrm{O}\left(\sigma^{1 / 2} \mathrm{w}_{\mathrm{o}}\right)},
\end{aligned}
$$

10 "Very fast" in the relation to the longitudinal length of the slender cylinder; in fact, "very slowly" in the natural length scale d of the cross flow problem. 
where (3.1b) was used to estimate the order of magnitude of the $\mathrm{z}$ derivative and $\left\{\mathbf{u}_{0} ; \mathrm{p}_{\mathrm{o}}\right\} \cong \mathrm{O}\left(\sigma^{1 / 2}\right)$, see section (2). From the $\mathrm{w}_{\mathrm{o}}$ equation one obtains, at once, that

$$
\bullet \mathrm{w}_{\mathrm{o}} \cong \mathrm{O}(\sigma) \text {, }
$$

showing that the longitudinal perturbed velocity $\mathrm{w}_{\mathrm{o}}$ is, as expected, of smaller order than the perturbed cross-flow $\mathbf{u}_{0}$. Furthermore, if terms of order $\sigma^{2}$ are disregarded, as before, it is possible to check from (3.2a) that the longitudinal velocity $\mathrm{w}_{\mathrm{o}}$ does not affect the (dynamic) $\mathbf{u}_{\mathrm{o}}$-equation: the longitudinal flow affects the $2 \mathrm{D}$ solution $\mathbf{u}_{\mathrm{o}}$ only through the mass conservation equation, with a term of order $\mathrm{O}\left(\sigma^{3 / 2}\right)$, the same order of magnitude of the longitudinal diffusion in the $\mathbf{u}_{\mathrm{o}}$-equation. One is left, thus, with the equation

$$
\begin{aligned}
& \bullet \frac{\partial \mathbf{u}_{\mathrm{o}}}{\partial \mathrm{t}}+\left(\mathbf{u}_{\mathrm{e}} \cdot \nabla\right) \mathbf{u}_{\mathrm{o}}+\left(\mathbf{u}_{\mathrm{o}} \cdot \nabla\right) \mathbf{u}_{\mathrm{e}}+\left(\mathbf{u}_{\mathrm{o}} \cdot \nabla\right) \mathbf{u}_{\mathrm{o}}-\frac{1}{\mathrm{Re}} \nabla^{2} \mathbf{u}_{\mathrm{o}}+\nabla \mathrm{p}_{\mathrm{o}}=\underbrace{\frac{1}{\operatorname{Re} \frac{\partial^{2} \mathbf{u}_{\mathrm{o}}}{\partial \mathbf{z}^{2}}}}_{\mathrm{O}\left(\sigma^{3 / 2}\right)} ; \\
& \qquad \underbrace{\frac{\partial \mathrm{w}_{\mathrm{o}}}{\partial \mathrm{t}}+\left(\mathbf{u}_{\mathrm{e}} \cdot \nabla\right) \mathrm{w}_{\mathrm{o}}-\frac{1}{\mathrm{Re}} \nabla^{2} \mathrm{w}_{\mathrm{o}}}_{\mathrm{O}(\sigma)}=-\underbrace{\frac{\partial \mathrm{p}_{\mathrm{o}}}{\partial \mathrm{z}}}_{\mathrm{O}(\sigma)} ; \\
& \bullet \nabla \cdot \mathbf{u}_{\mathrm{o}}=-\underbrace{\frac{\partial \mathrm{w}_{\mathrm{o}}}{\partial \mathrm{z}}}_{\mathrm{O}\left(\sigma^{3 / 2}\right)},
\end{aligned}
$$

that defines, with an error factor $\left[1+\mathrm{O}\left(\sigma^{2}\right)\right]$, an asymptotic approximation for $\mathbf{u}_{(3 \mathrm{~d})}(\mathbf{x}, \mathrm{z}, \mathrm{t})$.

Equation (3.2c) is, in some sense, standard in existing "slender bodies theories": the 2D structure, represented by the dynamic $\mathbf{u}_{0}$ equation, is not spoiled by the longitudinal velocity $\mathrm{w}_{\mathrm{o}}$, the influence of this parcel appearing only in an oblique way in the problem. In fact, a "spontaneous" 3D perturbation on the cross-flow introduces a pressure gradient that forces a longitudinal flow $\mathrm{w}_{\mathrm{o}}$ and only then, through mass conservation, the 3D perturbation feeds back the 2D original equation: as in the well known Lifting Line Theory, the three-dimensionality, represented by the "sidewash" $\mathrm{w}_{\mathrm{o}}$, affects essentially the kinematics of the 2D flow. Furthermore, the pressure gradient, and so the "sidewash", is proportional to $\partial \mathrm{A} / \partial \mathrm{z}$ and then $\nabla \cdot \mathbf{u}_{0} \propto \partial^{2} \mathrm{~A} / \partial \mathrm{z}^{2}$ : this correction on the cross-flow is added to the straight diffusion term, proportional to $\partial^{2} \mathbf{u}_{\mathrm{o}} / \partial \mathrm{z}^{2}$, to produce the Ginzburg coefficient $\gamma=\gamma_{\mathrm{R}}+\mathrm{i} \gamma_{\mathrm{I}}$. The influence of the "sidewash" on the final equation is thus twofold: first, it gives rise to a longitudinal diffusion, proportional to $\nabla^{2} \mathrm{w}_{\mathrm{o}}$, that together with the cross-flow diffusion $\partial^{2} \mathbf{u}_{\mathrm{o}} / \partial \mathrm{z}^{2}$ determines $\gamma_{\mathrm{R}}$; second, the longitudinal flow $\mathrm{w}_{\mathrm{o}}$ introduces a kind of "compressibility" for the cross-flow $\mathbf{u}_{\mathrm{o}}$ $\left(\nabla \cdot \mathbf{u}_{\mathrm{o}} \propto \partial^{2} \mathrm{~A} / \partial \mathrm{z}^{2}\right)$ and an "acoustic wave equation" must be expected then, described here by the "longitudinal wave operator" $\partial \mathrm{A} / \partial \mathrm{t}-\mathrm{i} \gamma_{\mathrm{I}} \partial^{2} \mathrm{~A} / \partial \mathrm{z}^{2}=0$ with a dispersion relation $\omega+\gamma_{\mathrm{I}} \mathrm{k}^{2}=0$. If the nonlinear term $i \mu_{\mathrm{I}}|\mathrm{A}|^{2} \mathrm{~A}$ is added to this wave operator one obtains the cubic Schrödinger equation $\mathrm{i} \partial \mathrm{A} / \partial \mathrm{t}+\gamma_{\mathrm{I}} \partial^{2} \mathrm{~A} / \partial \mathrm{z}^{2}-\mu_{\mathrm{I}}|\mathrm{A}|^{2} \mathrm{~A}=0$, a conspicuous presence in the study of nonlinear dispersive wave systems, see Whitham (1974). In what follows the discrete solution of (3.2c) will be defined and discussed.

\section{The "Sidewash" and Mass Conservation}

One starts by considering the $\mathrm{w}_{\mathrm{o}}$-equation, forced by the term $\partial \mathrm{p}_{\mathrm{o}} / \partial \mathrm{z}$; to leading order one has (see (2.30d) using $\left.\mathrm{p}_{11}(\mathbf{x}) \Leftrightarrow \mathbf{P}_{11}\right)$

$$
-\frac{\partial \mathrm{p}_{\mathrm{o}}}{\partial \mathrm{z}}=-\left[\mathrm{p}_{11}(\mathbf{x}) \frac{\partial \mathrm{A}}{\partial \mathrm{z}} \mathrm{e}^{\mathrm{i} \omega \mathrm{t}}+\left(^{*}\right)\right]+\mathrm{O}\left(\sigma^{3 / 2}\right),
$$

and thus writing $\mathrm{w}_{\mathrm{o}}(\mathbf{x}, \mathrm{z}, \mathrm{t})$ in the form

$$
\bullet \mathrm{w}_{\mathrm{o}}(\mathbf{x}, \mathrm{z}, \mathrm{t})=\left[\mathrm{w}_{11}(\mathbf{x}) \cdot \frac{\partial \mathrm{A}}{\partial \mathrm{z}} \mathrm{e}^{\mathrm{i} \omega \mathrm{t}}+\left(^{*}\right)\right],
$$

the following equation for $\mathrm{w}_{11}(\mathbf{x})$ can be obtained ${ }^{11}$ :

$$
\bullet i \omega \mathrm{w}_{11}(\mathbf{x})+\left(\mathbf{u}_{\mathrm{e}} \cdot \nabla\right) \mathrm{w}_{11}(\mathbf{x})-\frac{1}{\mathrm{Re}} \nabla^{2} \mathrm{w}_{11}(\mathbf{x})=-\mathrm{p}_{11}(\mathbf{x}) \cdot
$$

Taking the same mesh used to discretize both the velocity and pressure fields in section (2), namely, assuming

$$
\begin{aligned}
& \mathrm{w}_{11}(\mathbf{x})=\sum_{\mathrm{j}=1}^{\mathrm{N}} \mathrm{W}_{11, \mathrm{j}} \cdot \mathrm{h}_{\mathrm{j}}(\mathbf{x}) ; \mathbf{W}_{11}=\left\{\mathrm{W}_{11, \mathrm{j}}\right\} ; \\
& \mathrm{p}_{11}(\mathbf{x})=\sum_{\alpha=1}^{\mathrm{e}} \mathrm{P}_{11, \alpha} \cdot \mathrm{t}_{\alpha}(\mathbf{x}) ; \mathbf{P}_{11}=\left\{\mathrm{P}_{11, \alpha}\right\},
\end{aligned}
$$

and introducing the matrices $\left\{\mathbf{m} ; \mathbf{k} ; \mathbf{R}_{\mathrm{w}}\right\}$ by the expressions

$$
\begin{aligned}
& \int_{\mathrm{R}} \mathrm{w}_{11}(\mathbf{x}) \cdot \delta \mathrm{w}(\mathbf{x}) \mathrm{dR}=\delta \mathbf{W}^{\mathrm{t}} \cdot \mathbf{m} \cdot \mathbf{W}_{11} ; \int_{\mathrm{R}} \mathrm{p}_{11}(\mathbf{x}) \cdot \delta \mathrm{w}(\mathbf{x}) \mathrm{dR}=\delta \mathbf{W}^{\mathrm{t}} \cdot \mathbf{R}_{\mathrm{w}} \cdot \mathbf{P}_{11} ; \\
& \int_{\mathrm{R}}\left[\left(\mathbf{u}_{\mathrm{e}} \cdot \nabla\right) \mathrm{w}_{11}(\mathbf{x}) \cdot \delta \mathrm{w}(\mathbf{x})+\frac{1}{\mathrm{Re}} \nabla \mathrm{w}_{11}(\mathbf{x}) \cdot \nabla(\delta \mathrm{w}(\mathbf{x}))\right] \mathrm{dR}=\delta \mathbf{W}^{\mathrm{t}} \cdot \mathbf{k} \cdot \mathbf{W}_{11},
\end{aligned}
$$

the following algebraic equation is obtained for the "nodal values vector" $\mathbf{W}_{11}$,

$$
\bullet(\mathrm{i} \omega \mathbf{m}+\mathbf{k}) \cdot \mathbf{W}_{11}=-\mathbf{R}_{\mathrm{w}} \cdot \mathbf{P}_{11}
$$

the non-singularity of (3.4a) being granted by the fact that all eigenvalues $\left\{\chi_{j} ; \mathrm{j}=1,2, . ., \mathrm{N}\right\}$ of the matrix $\mathbf{k}$ have necessarily ${ }^{12}$ negative real parts and $\operatorname{so}\left\{\chi_{\mathrm{j}} \neq \mathrm{i} \omega\right.$ all $\left.\mathrm{j}\right\}$. The continuity equation in its weak form reads

$$
\int_{\mathrm{R}} \delta \mathrm{p}(\mathbf{x})\left[\nabla \cdot \mathbf{u}_{\mathrm{o}}+\left(\mathrm{w}_{11}(\mathbf{x}) \frac{\partial^{2} \mathrm{~A}}{\partial \mathrm{z}^{2}} \mathrm{e}^{\mathrm{i} \omega \mathrm{t}}+(*)\right)\right] \mathrm{dR}=0,
$$

and the discrete form of this equation is given by

$$
\bullet \mathbf{R}^{\mathrm{t}} \cdot \mathbf{U}_{\mathrm{o}}=-\left(\mathbf{R}_{\mathrm{w}}^{\mathrm{t}} \cdot \mathbf{W}_{11} \cdot \frac{\partial^{2} \mathrm{~A}}{\partial \mathrm{z}^{2}} \mathrm{e}^{\mathrm{i} \omega \mathrm{t}}+(*)\right)
$$

If (3.4b) is multiplied on the left by $\mathbf{R}$ and the definition of the "conjugated Laplacian" $\nabla=\mathbf{R} \cdot \mathbf{R}^{\mathrm{t}}$ is used, one obtains for $\mathbf{U}_{\mathrm{o}}(\mathrm{t})$ the equation

$$
\bullet \nabla \cdot \mathbf{U}_{\mathrm{o}}=-\left(\mathbf{R} \cdot\left(\mathbf{R}_{\mathrm{w}}^{\mathrm{t}} \cdot \mathbf{W}_{11}\right) \cdot \frac{\partial^{2} \mathrm{~A}}{\partial \mathrm{z}^{2}} \mathrm{e}^{\mathrm{i} \omega \mathrm{t}}+\left(^{*}\right)\right),
$$

whose general solution can be written as (recall that $\left.\operatorname{Null}(\nabla)=\mathrm{J}_{\mathrm{s}}\right)$

11 For simplicity, homogeneous boundary condition is assumed on $\partial \mathrm{R}$. Others boundary conditions could be used instead but this simple one is reasonable and easier from a more technical point of view.

12 The parcel proportional to ue in (3.3d) leads to an anti-symmetric matrix while the one proportional to $1 / \mathrm{Re}$ leads to a symmetric positive definite matrix. From this it follows at once that Real $\chi<0$ if $(\chi m+k) \cdot X=0$. 


$$
\bullet \mathbf{U}_{\mathrm{o}}(\mathrm{t})=\mathbf{T} \cdot \mathbf{q}_{\mathrm{o}}(\mathrm{z}, \mathrm{t})-\left[\mathbf{C} \cdot \frac{\partial^{2} \mathrm{~A}}{\partial \mathrm{z}^{2}} \mathrm{e}^{\mathrm{i} \omega \mathrm{t}}+\left(^{*}\right)\right]
$$

with $\mathbf{C} \in \mathrm{G}_{\mathrm{r}}$ being the unique (within $\mathrm{G}_{\mathrm{r}}$ ) solution of

$$
\bullet \nabla \cdot \mathbf{C}=\mathbf{R} \cdot\left(\mathbf{R}_{\mathrm{w}}^{\mathrm{t}} \cdot \mathbf{W}_{11}\right) \in \mathrm{G}_{\mathrm{r}} .
$$
(2.9b))

Notice that $\mathbf{C}$ can be defined by the sum of the series (see

$$
\bullet \mathbf{C}=\frac{1}{\kappa_{\mathrm{r}}} \sum_{\mathrm{n}=0}^{\infty} \nabla_{1}^{\mathrm{n}} \cdot \mathbf{R} \cdot\left(\mathbf{R}_{\mathrm{w}}^{\mathrm{t}} \cdot \mathbf{W}_{11}\right),
$$

where (3.5d) has again an "almost geometric structure" and its convergence can be thus accelerated by Shanks Transformation, see (2.15c).

\section{The Ginzburg-Landau Equation (GLE)}

The discrete form of the $\mathbf{u}_{\mathrm{o}}$-equation in (3.2c) is given by

$\delta \mathbf{U}^{\mathrm{t}} \cdot\left\{\mathbf{M} \cdot \dot{\mathbf{U}}_{\mathrm{o}}+\mathbf{K} \cdot \mathbf{U}_{\mathrm{o}}+\mathbf{N}\left(\mathbf{U}_{\mathrm{o}}\right) \cdot \mathbf{U}_{\mathrm{o}}-\mathbf{R} \cdot \mathbf{P}_{\mathrm{o}}\right\}=\delta \mathbf{U}^{\mathrm{t}} \cdot\left(\frac{1}{\operatorname{Re}} \mathbf{M} \cdot \mathbf{T} \cdot \mathbf{E} \cdot \frac{\partial^{2} \mathrm{~A}}{\partial \mathrm{z}^{2}} \mathrm{e}^{\text {iot }}+\left(*^{*}\right)\right)(3.6 \mathrm{a})$

where the leading order term

$$
\mathbf{U}_{\mathrm{o}}(\mathrm{z}, \mathrm{t})=\left[\mathbf{T} \cdot \mathbf{E} \mathrm{A}(\mathrm{z}, \mathrm{t}) \mathrm{e}^{\mathrm{i} \omega \mathrm{t}}+(*)\right] \cong \mathrm{O}\left(\sigma^{1 / 2}\right)
$$

was used in the right side of (3.2c). Placing now (3.5b) into (3.6a) one obtains, after projecting into the solenoidal sub-space, the equation

$$
\begin{aligned}
& \mathbf{M}_{\mathrm{s}} \cdot \dot{\mathbf{q}}_{\mathrm{o}}+\mathbf{K}_{\mathrm{s}} \cdot \mathbf{q}_{\mathrm{o}}-\left[\left(\mathbf{C}_{\mathrm{s}}+\frac{1}{\operatorname{Re}} \mathbf{M}_{\mathrm{s}} \cdot \mathbf{E}\right) \frac{\partial^{2} \mathrm{~A}}{\partial \mathrm{z}^{2}} \mathrm{e}^{\mathrm{i} \omega \mathrm{t}}+\left(^{*}\right)\right]+\mathbf{N}_{\mathrm{s}}\left(\mathbf{q}_{\mathrm{o}}\right) \cdot \mathbf{q}_{\mathrm{o}}=\mathbf{0} \\
& \mathbf{C}_{\mathrm{s}}=\mathbf{T}^{\mathrm{t}} \cdot(\mathrm{i} \omega \mathbf{M}+\mathbf{K}) \cdot \mathbf{C},
\end{aligned}
$$

whose asymptotic solution (2.25a) has an amplitude $\mathrm{A}(\mathrm{z}, \mathrm{t})$ that satisfies the GLE (3.1a) with

$$
\bullet \gamma=\gamma_{\mathrm{R}}+\mathrm{i} \gamma_{\mathrm{I}}=\mathbf{E}_{\mathrm{a}}^{\mathrm{t}} \cdot \mathbf{C}_{\mathrm{s}}+\frac{1}{\mathrm{Re}},
$$

since $\mathbf{E}_{\mathrm{a}}{ }^{\mathrm{t}} \cdot \mathbf{M} \cdot \mathbf{E}=1$. The remaining terms for the velocity and pressure fields are given by $(2.29 \mathrm{a}) ;(2.30 \mathrm{~b}, \mathrm{c}, \mathrm{d})$, adding the pressure parcels proportional to $\partial^{2} \mathrm{~A} / \partial \mathrm{z}^{2}$. The attention will be turned next to a more detailed analysis of GLE.

\section{GLE: Boundary Conditions and Wave-Like Limit Cycles}

If the cylinder's span is defined in the interval $-l \leq \mathrm{z} \leq l$ boundary conditions must be imposed at the cylinder ends $\mathrm{z}= \pm l$, one in each extremity. Using again the notation $\{\mathbf{e}(\mathbf{x}) \Leftrightarrow \mathbf{T} \cdot \mathbf{E}$; $\left.\mathrm{w}_{11}(\mathbf{x}) \Leftrightarrow \mathbf{W}_{11}\right\}$ the velocity field can be written, to leading order, in the form

$$
\mathbf{u}_{(3 \mathrm{D})}(\mathbf{x}, \mathbf{z , t})=\mathbf{u}_{\mathrm{e}}(\mathbf{x})+\left[(\underbrace{\mathrm{A}(\mathrm{z}, \mathrm{t}) \cdot \mathbf{e}(\mathbf{x})}_{\mathbf{u}_{0}(\mathbf{x}, \mathrm{z}, \mathrm{t})}+\underbrace{\frac{\partial \mathrm{A}}{\partial \mathbf{z}}(\mathrm{z}, \mathrm{t}) \cdot \mathrm{w}_{11}(\mathbf{x}) \mathbf{k}}_{\mathrm{w}_{\mathrm{o}}(\mathbf{x}, \mathrm{z}, \mathrm{t})}) \mathrm{e}^{\mathrm{i} \omega \mathrm{t}}+\left(^{*}\right)\right],(3.8 \mathrm{a})
$$

13 Namely, a larger cylinder is smoothly fitted to the bottom end, increasing locally the cross-flow and creating a bottom end condition similar to the one found at the free surface; see Khalak \& Williamson (1996) 
that reduces to the condition (i) in ( $3.8 \mathrm{~b})$ when $\mathrm{k}_{0}=0$ (2D solution): as a matter of fact, the "end-cylinder technique" was introduced just to create conditions to favor the parallel shedding on a cylinder in a water channel, see again Khalak \& Williamson (1996), since it forces, apparently, the condition $\partial \mathrm{A} / \partial \mathrm{z} \approx 0$ at the bottom end. Obviously, more assertive statements about some features of the solutions can only be done by direct numerical simulation of (3.1a) but it is felt that this simple stability analysis helps to focus some relevant issues.

\section{Spanwise Variation of Geometry and Current}

So far the analyzes was restricted to the simple uniform flow along a cylinder - being more precise, all empirical and numerical evidences commented here are related specifically to the flow around circular cylinders - although one should be concerned, from a more practical point of view, with problems where the cross section geometry and the incident current changes along the span, both in intensity and in direction. Relevant examples are the flow around a tapered cylinder, used to emulate a current variation along the span, the flow around a circular cylinder with strakes, very important from a practical point of view, or else the change of the of the incident flow direction along depth, a situation usually encountered in Ocean Engineering. In all these examples the unstable mode $\mathbf{E}_{\mathrm{U}}$ changes in the $\mathrm{z}$-direction and so it does the sectional pressure field $\mathbf{P}_{11}$ that forces the longitudinal flow $\mathrm{w}_{\mathrm{o}}(\mathbf{x}, \mathrm{z}, \mathrm{t})$, see $(2.30 \mathrm{~b}, \mathrm{c}, \mathrm{d})$; writing, as before, $\mathrm{p}_{11}(\mathbf{x}, \mathrm{z}) \Leftrightarrow \mathbf{P}_{11}(\mathrm{z})$, one has that $\partial \mathrm{p}_{11} / \partial \mathrm{z} \cong \mathrm{O}\left(\mathrm{p}_{11} / l_{\mathrm{g}, \mathrm{c}}\right)$, where $l_{\mathrm{g}, \mathrm{c}}$ is the length scale for the longitudinal variation of both the geometry and the current, and the question one intends to answer is the following: how small can be $l_{\mathrm{g}, \mathrm{c}}$ in order the GLE (3.1a) remains valid, with the same error factor of the form $\left[1+\mathrm{O}\left(\sigma^{2}\right)\right]$, even in the presence of these variations? Obviously, the basic parameters should then change (weakly) with $\mathrm{z}$ - namely, $\{\omega=\omega(\mathrm{z}) ; \sigma=\sigma(\mathrm{z}) ; \mu=\mu(\mathrm{z}) ; \gamma=\gamma(\mathrm{z})\}$ - but the structure of (3.1a) would remain the same and so the $2 \mathrm{D}$ expressions used to compute them.

To answer such question one must recall that the threedimensionality was forced by the pressure gradient that, to leading order, is given by

$$
\frac{\partial p}{\partial z}(\mathbf{x}, z, t)=\left[\left(\frac{\partial A}{\partial z} p_{11}+A \frac{\partial p_{11}}{\partial z}\right) e^{i \omega t}+\left(^{*}\right)\right]+O\left(\sigma^{3 / 2}\right)
$$

and observing that to retain the final error $\mathrm{O}\left(\sigma^{2}\right)$ only the term of order $\mathrm{O}(\sigma)$ must be kept in $(3.14 \mathrm{a})$, the variation $\partial \mathrm{p}_{11} / \partial \mathrm{z}$ could be ignored if it is of order $\mathrm{O}(\sigma)$ or smaller: in this case the term $\mathrm{A} \cdot \partial \mathrm{p}_{11} / \partial \mathrm{z}$ would be of order $\mathrm{O}\left(\sigma^{3 / 2}\right)$ or smaller, since $\mathrm{A} \cong \mathrm{O}\left(\sigma^{1 / 2}\right)$, and the variation of the geometry and/or the incident flow direction would appear at most at the order $\mathrm{O}\left(\sigma^{2}\right)$ and can, thus, be disregarded. It turns out that (3.1a) remains correct, with the sectional parameters $\{\omega(\mathrm{z}) ; \sigma(\mathrm{z}) ; \mu(\mathrm{z}) ; \gamma(\mathrm{z})\}$, whenever the length scale $l_{\mathrm{g}, \mathrm{c}}$ is so large that $\left(\partial \mathrm{p}_{11} / \partial \mathrm{z} \cong \mathrm{O}\left(\mathrm{p}_{11} / l_{\mathrm{g}, \mathrm{c}}\right)\right)$

$$
\frac{\partial \mathrm{p}_{11}}{\partial \mathrm{z}} \leq \mathrm{O}(\sigma) \Leftrightarrow l_{\mathrm{g}, \mathrm{c}} \geq \mathrm{O}\left(\frac{d}{\sigma}\right)
$$

As seen in (2.31), empirical evidences suggest that $\sigma \approx 0.20$ and so $l_{\mathrm{g}, \mathrm{c}} \geq \mathrm{O}(5 d)$, the fastest change in z-direction within the context of GLE being defined by the relation $l_{\mathrm{g}, \mathrm{c}} \approx 5 d$. It is a matter of curiosity to observe that $5 d$ is a typical length scale for most of the "suppressions devices" used to mitigate (or eliminate) VIV; for instance, this is a typical value for the helicoidal pitch of the strakes or for the wavelength of the wavy cylinder analyzed by Bearman (2000).

\section{Conclusion}

In the present paper a consistent asymptotic approximation for the flow around a slender cylinder was developed, leading to the Ginzburg-Landau equation. The theory is based on an assumption concerning the behavior of the eigenvalues related to a $2 \mathrm{D}$ perturbation on the steady $2 \mathrm{D}$ solution $\mathbf{u}_{\mathrm{e}}(\mathbf{x})$; it states that there exists only one unstable mode with eigenvalue $\lambda_{1}=\sigma+i \omega$ and, furthermore, that $\{\omega \neq 0 ; 0<\sigma<<1\}$, see (2.21).

Both conditions are satisfied in the vicinity of the Hopf bifurcation at $\mathrm{Re} \approx \mathrm{Re}_{\mathrm{cr}} \cong 45$ but to obtain the desired approximation some more technical results were needed. First, the "wake impedance" was introduced, by considering properly the flow in the wake and determining then how the wake "resists" to the flow within the finite fluid region that is actually discretized; second, by projecting the discrete flow equations into the solenoidal and gradient sub-spaces it has been possible, using some standard results in Linear Algebra, to show not only the inner consistency of the model but also to obtain the coefficients of the Ginzburg-Landau equation. In particular, the Ginzburg coefficient $\gamma=\gamma_{R}+i \gamma_{I}$ was analyzed, where the "diffusive feature" of the real part $\gamma_{R}$ was elaborated and also the "wave feature" of $\gamma_{\mathrm{I}}$ was established, once it is related to the "compressibility" of the cross-flow, namely, to the work done by the cross-flow pressure field on the divergence of the cross-flow velocity field.

The final goal of the on-going research is to address the VIV problem, of considerable importance in some Ocean Engineering applications, mainly in the analysis of the "risers" of a floating production system. This problem has been tentatively addressed, with a relative success, by the so-called "phenomenological models", where the flow is simply described by a Van der Pol oscillator with coefficients inferred from some experimental results. The situation here is not very much different, at least from the operational point of view, to the usual approach related to GinzburgLandau equation (GLE), once this "model" is fitted externally to the problem and the coefficients are then inferred also by some experimental (or numerical) results. But there is a conceptual difference, at least in its origin, in both approaches: GLE was thought to be valid only in the vicinity of $\mathrm{Re}_{\mathrm{cr}}$ (although it has been used far beyond it, at least up to the range $\mathrm{Re} \approx 300$ ) while the Van der Pol model was aimed, from its very first motivation, to deal with the experiments on VIV, where $10^{3}<\mathrm{Re}<10^{4}$ roughly speaking. Observing that the Van der Pol model assumes, implicitly, that only one mode is unstable with a "negative damping" $\sigma<<1$, one may be tempted to conclude, based on the relatively good predictive ability of these "phenomenological models" and also on the already proposed extensions of GLE to the range $\operatorname{Re} \approx 300$, that the underlying assumption of the present asymptotic theory holds, in fact, in a much broader range of Reynolds numbers than foreseen a priori.

This conjecture can be raised to the status of a hypothesis in the mathematical development and, with it, to extend consistently the GLE to the "whole" range of Re; afterwards, by looking to the actual numerical results one can confirm (or not) this hypothesis. The present theory does not seem to be at odds, thus, with tradition in physical science: indeed, given a set of a somewhat disperse results and observations, all of them related however to the conspicuous oscillatory behavior of the phenomenon, they can be gathered by means of an assumption, synthesized by (2.21), that places all them in an unique framework, namely, the GLE in a "wide range" of Reynolds numbers. Furthermore, as stated above, this theory brings in its lay out the possibility to be refuted, once the basic assumption can be checked directly by means of (numerical) experiments; or, from a more practical point of view, it allows one 
to determine precisely how "wide" can be the range of Reynolds numbers covered by it.

The route to be followed next, in this on-going research, is thus clear: first, to obtain numerical results that could possibly confirm assumption (2.21) in a certain range of Re; second, to obtain an extension of the GLE equation that can deal also with an oscillating cylinder; third, to compare the VIV predictions obtained from this extended model with the existing experimental results. The hope is that they will provide consistent results but, anyway, one has here at least a consistent theory in a relatively large vicinity of $\mathrm{Re}_{\mathrm{cr}}$, what may have an interest in itself.

\section{References}

Albarède,P. \& Provansal,M. \& Boyer,L. (1990): "Modélisation par l'équation Ginzburg-Landau du sillage tridimensionnel d'un obstacle allongé", C.R.Acad.Sci.Paris Sér.II 310,459;

Albarède,P. \& Monkewitz,P.A. (1992): "A model for the formation of oblique shedding and "chevron" patterns in cylinder wakes", Phys. Fluids A, Vol.4, No.4, pp. 744-756;

Albarède, P. \& Provansal,M. (1995): “Quase-periodic cyinder wakes and the Ginzburg-Landau model”, J. Fluid Mech., vol. 291, pp.191-222;

Aranha, J.A.P. (2003): "On the "div-stability" condition in discrete Navier-Stokes equation”, submitted;

Bathe,K.J. (1996): “Finite Element Procedures”, Prentice Hall;

Bearman,P.W. (2000): Private Communication;

Bender,C.M. \& Orszag,S.A. (1978): "Advanced Mathematical Methods

for Scientists and Engineers", McGraw-Hill Book Company;

Benjamin,T.B. \& Feir, J.E. (1967): "The desintegration of wave trains on deep water", J. Fluid Mech. 27, 417;

Bishop,R.E.D. \& Hassan,A.Y. (1964): "The lift and drag forces on a circular cylinder in a flowing field”, Proc. Roy. Soc. (London), Ser.A, 277,pp. 51-75;

Ginzburg,V.L. \& Landau,D. (1950): “On the theory of superconductivity”, Zh. Eksp. Teor. Fiz. 20,1064;
Gunzburger,M.D. (1985): "Finite Element Methods for Viscous Incompressible Flows", Academic Press Inc.;

Henderson,R.D. (1997): "Nonlinear dynamics and pattern formation in turbulrnt wake transition", J. of Fluid Mech., vol.352, pp.65-112;

Huerre,P. \& Monkewitz,P.A. (1990): "Local and global instabilities in spatially developing flows", Ann. Ver. Fluid Mech., 32, pp. 473-537;

Khalak,A. \& Wiliamson,H.K. (1996): "Dynamics of a hydroelastic cylinder with very low mass and damping", J. of Fluid and Structures, vol. 10, pp.455-472;

Iwan,W.D. \& Blevins,R.D. (1975): “A model for vortex-induced oscillation of structures", J. Appl. Mech., 41, pp.581-586;

Ladyzenskaja,O.A. (1969): "The Mathematical Theory of Viscous Incompressible Flow", Gordon and Breach Science Publishers;

Lehoucq,R.B. \& Sorensen,D.C. \& Yang,C. (1997): “ARPACK User's Guide: Solution of Large Scale Eigenvalue Problems with Implicit Restarted Arnoldi Methods", arpack@caam.rice.edu;

Leweke,T. \& Provansal,M. (1994): "Model for transition in bluff body wakes", Physical Review Letters, V.72, N.20, pp.3174-3177;

Monkewitz,P.A. (1996): "Modeling of self-excited wake oscillations by amplitude equations", Experimental Thermal and Fluid Science, 12, pp.175183 ;

Monkewitz,P.A. \& Williamson,C.H.K. \& Miller,G.D. (1996): "Phase dynamics of Kármán vortices in cylinder wakes", Phys. Fluids 8(1),pp.9196;

Noack,B.R. \& Eckelmann,H. (1994): “A global stability of the steady and periodic cykinder wake", J. Fluid Mech., vol.270, pp.297-330;

Provansal,M. \& Mathis,C. \& Boyer,L. (1987): "Bérnard-von Kárman instability: transient and forced regimes", J. Fluid Mech., 182, pp. 1-22;

Toebes,G.H. (1969): “The unsteady flow and wake near an oscillating cylinder", J.Basic Eng., 91,493;

Van Dyke,M. (1982): “An Album of Fluid Motion”, The Parabolic Press;

Whitham,G.B. (1974): "Linear and Nonlinear Waves", John Wiley \& Sons, N.Y.;

Zakharov,V.E. \& Shabat,A.B. (1972): "Exact theory of two-dimensional self focusing and one dimensional self modulation of waves in nonlinear media", Soviet Physics J.E.T.P. 34, pp.62-69. 